\title{
Analysis of a Galerkin-characteristic finite element method for convection-diffusion problems in porous media
}

\section{L'analyse d'une méthode semi-lagrangienne et éléments finis pour les problémes de convection-diffusion en milieux poreux}

\author{
Loubna Salhi ${ }^{1}$, Mofdi El-Amrani ${ }^{2}$, and Mohammed Seaid ${ }^{3,4}$ \\ ${ }^{1}$ Laboratory Modeling Simulation \& Data Analysis, University Mohammed VI Polytechnic, Benguerir, Morocco \\ loubna.salhi@um6p.ma \\ ${ }^{2}$ Mathematics and Applications Laboratory, FST, Abdelmalek Essaadi University, Tangier, Morocco \\ mofdi.elamrani@gmail.com \\ ${ }^{3}$ Department of Engineering, University of Durham, South Road, Durham DH1 3LE, UK \\ m.seaid@durham.ac.uk \\ ${ }^{4}$ International Water Research Institute, University Mohammed VI Polytechnic, Benguerir, Morocco
}

RÉSUMÉ. L'objectif de ce travail est l'étude d'une méthode de résolution numérique par éléments finis semilagrangiennes afin de résoudre les problémes évolutifs de convection-diffusion issus des milieux poreux. La méthode proposée permet d'utiliser une approximation par éléments finis d'ordre égal pour toutes les solutions du probléme. En outre, la condition standard de Courant-Friedrichs-Lewy est assouplie avec le traitement lagrangien des termes de convection, et les erreurs de troncature sont réduites dans la partie diffusion-réaction du probléme. Dans cette étude, une analyse de la convergence et de la stabilité de la méthode proposée est aussi présentée, ainsi que les estimations des erreurs dans la norme $L^{2}$ dérivées pour toutes les solutions numériques. Les tests numériques sont illustrées par quelques exemples afin de vérifier les estimations théoriques et de démontrer la grande précision et l'efficacité de la méthode proposée.

ABSTRACT. We present a Galerkin-characteristic finite element method for the numerical solution of time-dependent convection-diffusion problems in porous media. The proposed method allows the use of equal-order finite element approximations for all solutions in the problem. In addition, the standard Courant-Friedrichs-Lewy condition is relaxed with the Lagrangian treatment of convection terms, and the time truncation errors are reduced in the diffusion-reaction part. Analysis of convergence and stability of the proposed method is also investigated in this study and error estimates in the $L^{2}$-norm are established for the numerical solutions. Numerical performance of the method is examined using two examples to verify the theoretical estimates and to demonstrate the high accuracy and efficiency of the proposed Galerkin-characteristic finite element method.

MOTS-CLÉS. Problémes de convection-diffusion, Équation de Darcy, Milieux poreux, Méthode semi-lagrangienne, Éléments finis, Estimation des erreurs a priori.

KEYWORDS. Convection-diffusion problems, Darcy equation, Porous media, Galerkin-characteristic method, Finite elements, A priori error estimates.

\section{Introduction}

Convection-diffusion problems in porous media occur in several physical applications in fluid mechanics such as, petroleum reservoir engineering, geological sequestration of carbon dioxide, transport of water and contaminants in saturated zones and aquifers, heat explosion problems in the chemical industry, see $[3,16,17,29,49,31]$ among others. These problems involve many complex physical, chemical and fluid flow processes which are typically described in mathematical terms by coupling the mass conservation principle with the phenomenological Darcy's law, see for instance [4, 5]. Let $\Omega \subset \mathbb{R}^{d}$ $(d=2,3)$ be a bounded domain with Lipschitz continuous boundary $\Gamma$ and $[0, T]$ a given time interval. 
In the present work, we focus on a time-dependent convection-diffusion problem coupled with the Darcy equation. For all $(\boldsymbol{x}, t)$ in the domain $\Omega \times[0, T]$, the system of dimensionless equations reads

$$
\begin{aligned}
\nu(\Theta) \boldsymbol{u}+\nabla p & =\boldsymbol{f}(\Theta), & & \text { in } \Omega \times[0, T], \\
\nabla \cdot \boldsymbol{u} & =0, & & \text { in } \Omega \times[0, T], \\
\frac{D \Theta}{D t}-\kappa \nabla^{2} \Theta & =g(\boldsymbol{x}, t), & & \text { in } \Omega \times[0, T],
\end{aligned}
$$

In the Darcy equations (1a), $\boldsymbol{u}$ is the velocity field, $p$ the pressure, $\nu$ and $\boldsymbol{f}$ are the viscosity and the force density respectively which both depend on the fluid temperature $\Theta$. In the convection-diffusion equation (1b), $\kappa$ is the thermal diffusivity coefficient supposed to be a positive constant and $g$ an external source term, and

$$
\frac{D \Theta}{D t}=\frac{\partial \Theta}{\partial t}+\boldsymbol{u} \cdot \nabla \Theta
$$

is the material derivative which measures the rate of variation in the temperature $\Theta$ along the trajectories of the flow particles known by characteristic curves. In order for the mathematical problem to be well posed, the system (1a)-(1b) needs to be equipped with an initial condition

$$
\Theta(\boldsymbol{x}, 0)=\Theta_{0}(\boldsymbol{x}), \quad \text { in } \Omega,
$$

as well as given boundary conditions. Note that different types of boundary conditions may be adopted for equations (1a)-(1b) without major conceptual changes in the formulation. For simplicity in the presentation, homogeneous Dirichlet boundary conditions are considered for the pressure and temperature, while no-slip boundary conditions are applied for the velocity. Thus, the associated boundary conditions are given by

$$
\begin{aligned}
\boldsymbol{u} \cdot \boldsymbol{n} & =0, \\
\Theta & =0, \quad \text { on } \Gamma,
\end{aligned}
$$

where $\boldsymbol{n}$ is the unit outward normal vector on the boundary $\Gamma$. Note that other boundary conditions in (1e)-(1f) can also be used in this study without major conceptual modifications. The mathematical theory and existence of a weak solution for the problem (1) is presented in $[15,27]$ among others. The numerical resolution of this type of problems is still a challenging issue. Indeed, in the case of dominated convection, especially when the diffusion coefficient $\kappa$ reaches very low values, the convective term in (1b) could be a source of severe computational difficulties and nonphysical oscillations. Moreover, numerical solutions need an accurate approximation in order to resolve possible steep fronts and shocks, see for instance $[19,21,38,44]$. To deal with the difficulties generated by the presence of convective terms in the governing equations, it is possible to opt for numerical techniques based on Lagrangian methods, see for example $[13,26]$. However, the main disadvantage of these methods is the grid distortion drawback specially when characteristic curves are required to be computed for time-dependent velocity fields. Furthermore, the Eulerian finite element methods employ fixed grids and incorporate some upstream weighting in their formulations to stabilize the spatial discretization. These methods are easy to formulate and to implement. However, time truncation errors dominate their solutions and are subject to the Courant-Friedrichs-Lewy (CFL) stability conditions, which impose a sever restriction on the size of 
the time steps taken in the numerical simulations. Among the Eulerian methods we mention the streamline diffusion methods, the Petrov-Galerkin methods in addition to the the high resolution methods from computational fluid dynamics, in particular, the Godunov methods and the essentially non-oscillatory methods, see for example [32, 34, 47].

Modified method of characteristics also known in the meteorological community by semi-Lagrangian methods, are a particularly wise choice for the solution of many time-dependent convection-dominated equations. These Galerkin-characteristic methods have been successfully used in many fields such as oceanography, geoscience, meteorology for weather forecasting, and more general problems in fluid dynamics, see for instance $[1,39,40,45]$. The basic idea behind these methods is to convert the governing equations from an Eulerian formulation to a Galerkin-characteristic one in terms of the particle trajectories (or characteristic curves) associated with the total derivative (1c). The time derivative and the convection terms are combined as a directional derivative along the particles trajectories, leading to a characteristic time-stepping procedure. This procedure results in a substantial reduction in the computational cost and in the time truncation errors. Moreover, it offers the possibility of using time steps that exceed those allowed by the stability CFL condition for the conventional Eulerian methods. The Galerkin-characteristic methods have been subject of investigations in many references including $[6,19,20,21,36,37,38,42,43,44,46]$. In [19], the semi-Lagrangian method combined with a finite difference discretization has been studied and applied to convection-diffusion problems. In [20], the method has been used with a Galerkin finite element discretization. In addition, an $L^{2}$ interpolation procedure has also been developed by tracking the feet of the characteristic curves from the integration nodes. In [23, 25], the method has proved to be stable and accurate when used to study the thermal incompressible Navier-Stokes equations. Semi-Lagrangian methods have also been investigated for the simulation of natural and mixed convection flows in [21, 43], for tidal flows in [24], and for moving thermal fronts in porous media in [42]. A first-order Galerkin-characteristic method combined with the finite element discretization has been analyzed for the Navier-Stokes equations in [38]. It has been shown that the method is unconditionally stable provided the characteristics are transported by divergence-free velocity field. The case where the characteristics are transported by a discrete velocity field which is not divergence-free has also been studied in [46].

In this paper, we propose a Galerkin-characteristic finite element method for the numerical solution of the coupled convection-diffusion-Darcy equations (1). The main advantage of the proposed method lies in the use of unified finite element approximations for the spatial discretization of the Darcy problem. This class of finite elements has been well developed and analyzed in $[9,10,18]$, and has been shown to improve the accuracy for the solution of Darcy and Stokes problems. Indeed, unlike mixed finite elements for which approximation spaces with different orders are used for the spatial discretization, the unified finite element methods allow the same approximation spaces to be used for the pressure and velocity solutions. It has also been shown in $[9,10,18]$ that, when using an equal order interpolation, the method offers simple and uniform data structures and a reasonable accuracy. To the best of our knowledge, Galerkin-characteristic methods developed so far for flow and transport problems employ mixed finite element formulations. To this end, our objective is to investigate the unified finite element approach in the framework of Galerkin-characteristic solution of coupled convection-diffusion-Darcy problems. Another advantage of combining unified finite element discretizations with the Galerkincharacteristic is that the interpolation procedure at the characteristic feet can be performed using the same basis functions. Consequently, this reduces the computational cost related to the mesh generation 
and requires less implementation work than using the mixed finite element discretizations. Moreover, the proposed method is suitable for complex geometries, independent of the size and arrangement of the mesh elements, and can easily combine different polynomial orders of elements. These properties are very useful when coupling the Darcy flows to complex components to simulate problems, for example, in transport in porous media and multiphase flows. In the present work, a second-order accuracy is achieved for all solutions in the problem (1). In terms of analysis, we demonstrate the existence and uniqueness of solutions for the problem (1), present analysis of convergence and stability for the proposed method and thus, derive optimal a priori error estimates for the numerical solutions in the framework of $L^{2}$-theory. To validate the theoretical results and examine the performance of the Galerkin-characteristic finite element method, we perform several numerical tests. We consider a time-dependent coupled convection-diffusion Darcy problem with known analytical solution. The obtained results demonstrate good resolution with high accuracy and stability behaviors.

The paper is organized as follows. Notations, functional spaces and assumptions used for the analysis of stability and convergence are introduced in Section 2. Formulation of the Galerkin-characteristic unified finite element method used for solving the coupled Darcy-convection-diffusion equations (1) is presented in section 3. Analysis of stability and convergence along with a priori error estimates in the $L^{2}$ norm are established in Section 4. Some numerical experiments are presented in section 5. Concluding remarks are given Section 6.

\section{Preliminaries and assumptions}

In this section, we introduce the main notations and some approximation results that will be used throughout this paper. In what follows, we use the notation $w$ to denote the value of a generic scalar function, the boldface notation $\boldsymbol{w}$ to denote a vector valued function, and $K, K^{\prime}, K^{\prime \prime}, K 1, \ldots$ to denote generic positive constants whose values may change from one place to another but that remain independent of the mesh parameters. Spaces consisting of vector valued functions will be denoted in boldface. Let the notation $|\cdot|$ be the standard Euclidean norm in $\mathbb{R}^{d}(d=2,3)$. We denote by $C^{k}(\Omega), k \geq 1$ the class of functions whose partial derivatives of order at least $k$ are bounded and uniformly continuous in $\Omega$, whereas $C_{0}^{k}(\Omega)$ denotes the set of compactly supported functions contained in $C^{k}(\Omega)$. We introduce the Sobolev spaces $W^{m, p}(\Omega)$ for any integers $(m, p), m \geq 1$ and $1 \leq p \leq \infty$ as

$$
W^{m, p}(\Omega)=\left\{\boldsymbol{w} \in L^{p}(\Omega)^{d}: \quad D^{k} \boldsymbol{w} \in L^{p}(\Omega)^{d}, \quad \forall|k| \leq m\right\},
$$

where $k=\left(k_{1}, \ldots, k_{d}\right) \in \mathbb{N}^{d}$ is a multi-index of order $|k|=k_{1}+\cdots+k_{d}$, and the derivative operator $D^{k}$ is given by

$$
D^{k}=\frac{\partial^{|k|}}{\partial x_{1}^{k_{1}} \cdots \partial x_{d}^{k_{d}}}
$$

These spaces are equipped with the following norms

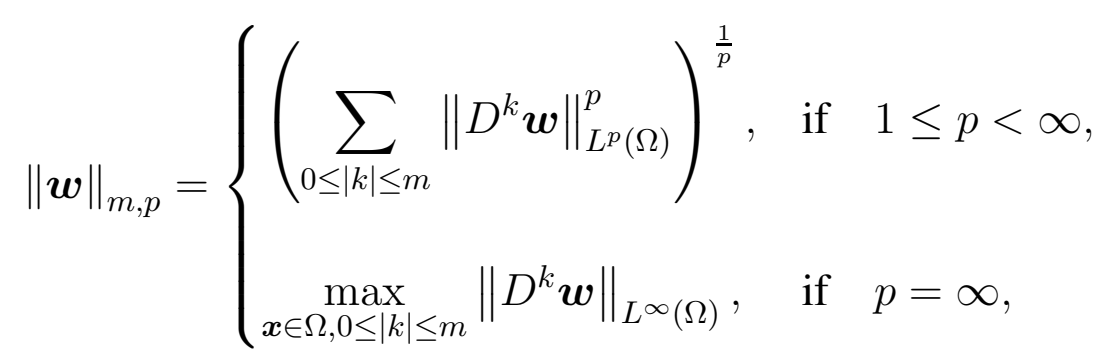


and the semi-norms

$$
|\boldsymbol{w}|_{m, p}=\left\{\begin{array}{l}
\left(\sum_{|k|=m}\left\|D^{k} \boldsymbol{w}\right\|_{L^{p}(\Omega)}^{p}\right)^{\frac{1}{p}}, \quad \text { if } 1 \leq p<\infty \\
\max _{\boldsymbol{x} \in \Omega,|k|=m}\left\|D^{k} \boldsymbol{w}\right\|_{L^{\infty}(\Omega)}, \quad \text { if } \quad p=\infty
\end{array}\right.
$$

In the special case $p=2$, the space $W^{m, 2}(\Omega)(m \geq 1)$ forms the Hilbert space $H^{m}(\Omega)$ which is the closure of $C^{\infty}(\Omega)$, while $H_{0}^{m}(\Omega)$ is the closure of $C_{0}^{\infty}(\Omega)$ with respect to the same norm. In particular, for $m=1$ we introduce the following spaces

$$
H^{1}(\Omega)=\left\{w \in L^{2}(\Omega): \quad \partial^{k} w \in L^{2}(\Omega), \quad \forall|k| \leq 1\right\}, \quad H_{0}^{1}(\Omega)=\left\{w \in H^{1}(\Omega):\left.\quad \boldsymbol{w}\right|_{\Gamma}=0\right\} .
$$

We define the space

$$
L^{2}(\Omega)=\left\{w: \Omega \longrightarrow \mathbb{R}: \quad \int_{\Omega} w^{2} d \Omega<\infty\right\}
$$

whose inner product and norm are denoted by

$$
(w, v)=\int_{\Omega} w v d \Omega \quad \text { and } \quad\|w\|_{L^{2}(\Omega)}=(w, w)^{\frac{1}{2}}, \quad \forall w, v \in L^{2}(\Omega),
$$

respectively. We also define the space $L_{0}^{2}(\Omega)$ of all square integrable functions with vanishing mean as

$$
L_{0}^{2}(\Omega)=\left\{w: \Omega \longrightarrow \mathbb{R}: \quad \int_{\Omega} w d \Omega=0\right\}
$$

We recall the standard spaces $\mathbf{H}(\operatorname{div}, \Omega)$ and $\mathbf{H}_{0}($ div,$\Omega)$

$$
\begin{aligned}
& \mathbf{H}(\operatorname{div}, \Omega)=\left\{\boldsymbol{w} \in\left(L^{2}(\Omega)\right)^{d}: \quad \operatorname{div}(\boldsymbol{w}) \in L^{2}(\Omega)\right\}, \\
& \mathbf{H}_{0}(\operatorname{div}, \Omega)=\left\{\boldsymbol{w} \in \mathbf{H}(\operatorname{div}, \Omega):\left.\quad(\boldsymbol{w} \cdot \boldsymbol{n})\right|_{\Gamma}=0\right\} .
\end{aligned}
$$

For any $1 \leq p \leq \infty$ and a Banach space $X$, we introduce the standard Bochner space $L^{p}(0, T ; X)$ consisting of all measurable functions $w(t, \boldsymbol{x})$ defined in $[0, T] \times \Omega$ for which

$$
\begin{aligned}
\int_{0}^{T}\|w\|_{X}^{p} d t<\infty, \quad \text { if } \quad 1 \leq p<\infty, \\
\operatorname{ess} \sup _{t \in[0, T]}\|w(\cdot, t)\|_{X}<\infty, \quad \text { if } \quad p=\infty
\end{aligned}
$$

The space $L^{p}(0, T ; X)$ is equipped with the norm

$$
\|w\|_{L^{p}(0, T ; X)}=\left\{\begin{array}{l}
\left(\int_{0}^{T}\|w\|_{X}^{p} d t\right)^{1 / p}, \quad \text { if } \quad 1 \leq p<\infty \\
\operatorname{ess} \sup _{t \in[0, T]}\|w(\cdot, t)\|, \quad \text { if } \quad p=\infty
\end{array}\right.
$$


Then, for the time discretization we divide the time interval $[0, T]$ into $N_{T}$ subintervals $\left[t_{n}, t_{n+1}\right]$ with length $\Delta t=t_{n+1}-t_{n}$ for $n=0,1, \ldots, N_{T}$. For the space discretization, let $\Omega_{h} \subset \bar{\Omega}=\Omega \cup \Gamma$ denotes a quasi-uniform partition of $\Omega$ into triangular finite elements $\mathcal{T}_{j}$ with the partition step $h$. The triangles $\mathcal{T}_{j}$ satisfy the following conditions:

(i) $\bar{\Omega}=\bigcup_{j=1}^{N e} \mathcal{T}_{j}$, where $N e$ is the number of elements of the partition $\Omega_{h}$.

(ii) If $\mathcal{T}_{i}$ and $\mathcal{T}_{j}$ are two different elements of the partition $\Omega_{h}$, then

$$
\mathcal{T}_{i} \cap \mathcal{T}_{j}= \begin{cases}P_{i j}, & \text { a mesh point, or } \\ \Gamma_{i j}, & \text { a common side, or } \\ \emptyset, & \text { empty set. }\end{cases}
$$

(iii) There exists a positive constant $\kappa$ such that for all $j \in\{1, \cdots, N e\}, \frac{d_{j}}{h_{j}}>\kappa\left(h_{j} \leq h\right)$, where $d_{j}$ is the diameter of the circle inscribed in $\mathcal{T}_{j}$ and $h_{j}$ is the largest side of $\mathcal{T}_{j}$.

The conforming finite element space for the temperature and pressure is defined as

$$
V_{h}=\left\{v_{h} \in C^{0}(\Omega):\left.\quad v_{h}\right|_{\mathcal{T}_{j}} \in P_{k}\left(\mathcal{T}_{j}\right), \quad \forall \mathcal{T}_{j} \in \Omega_{h}\right\},
$$

where $P_{k}\left(\mathcal{T}_{j}\right)$ is the space of complete polynomials of degree $k, k \geq 2$, on each element $\mathcal{T}_{j}$. We also define the conforming finite element space $\mathbf{V}_{h}=\left(V_{h}\right)^{d}$ for the velocity field. Hence, we formulate the finite element solutions to $\boldsymbol{u}^{n}(\boldsymbol{x}), p^{n}(\boldsymbol{x})$ and $\Theta^{n}(\boldsymbol{x})$ as

$$
\boldsymbol{u}_{h}^{n}(\boldsymbol{x})=\sum_{j=1}^{M} U_{j}^{n} \cdot \varphi_{j}(\boldsymbol{x}), \quad p_{h}^{n}(\boldsymbol{x})=\sum_{j=1}^{M} P_{j}^{n} \phi_{j}(\boldsymbol{x}), \quad \Theta_{h}^{n}(\boldsymbol{x})=\sum_{j=1}^{M} \mathcal{C}_{j}^{n} \phi_{j}(\boldsymbol{x}),
$$

with $U_{j}^{n}, P_{j}^{n}$ and $\mathcal{C}_{j}^{n}$ are the corresponding nodal values of $\boldsymbol{u}_{h}^{n}(\boldsymbol{x}), p_{h}^{n}(\boldsymbol{x})$ and $\Theta_{h}^{n}(\boldsymbol{x})$ respectively defined by $U_{j}^{n}=\boldsymbol{u}_{h}^{n}\left(\boldsymbol{x}_{j}\right), P_{j}^{n}=p_{h}^{n}\left(\boldsymbol{x}_{j}\right)$ and $\mathcal{C}_{j}^{n}=\Theta_{h}^{n}\left(\boldsymbol{x}_{j}\right)$, with $\left\{\boldsymbol{x}_{j}\right\}_{j=1}^{M}$ being the set of mesh points in the partition $\Omega_{h}$, and $\left\{\varphi_{j}\right\}_{j=1}^{M}=\left\{\left(\phi_{j}, \phi_{j}\right)\right\}_{j=1}^{M}$ and $\left\{\phi_{j}\right\}_{j=1}^{M}$ are the basis vectors and functions of $\mathbf{V}_{h}$ and $V_{h}$ respectively given by the Kronecker delta symbol.

For the variational formulation, we shall need the equal-order finite element pair $\left(\mathbf{S}_{h}, Q_{h}\right)$ to approximate the velocity and pressure solutions of the Darcy equations (1a), defined as

$$
\mathbf{S}_{h}=\mathbf{V}_{h} \cap \mathbf{H}_{0}(\operatorname{div}, \Omega) \quad \text { and } \quad Q_{h}=V_{h} \cap L_{0}^{2}(\Omega) .
$$

We also define the necessary finite element space $R_{h}$ to approximate the temperature solution of the convection-diffusion equation (1b) as

$$
R_{h}=V_{h} \cap H_{0}^{1}(\Omega) .
$$

Next, we announce the following assumptions:

ASSUMPTION 1. The velocity field $\boldsymbol{u}$ is assumed to satisfy

$$
\text { 1. } \boldsymbol{u}(\boldsymbol{x}, t) \in C^{0}\left(0, T ; W^{1, \infty}(\Omega)\right) \text {, }
$$


2. $\nabla \cdot \boldsymbol{u}(\boldsymbol{x}, t)=0$ in $\Omega$,

3. $\boldsymbol{u}(\boldsymbol{x}, t) \cdot \boldsymbol{n}=0$ on $\Gamma$.

AsSUMPTION 2. The temperature $\Theta$ satisfies

1. $\Theta \in L^{\infty}\left(0, T ; H^{m+1} \cap W^{m+1, \infty}(\Omega)\right)$,

2. $\frac{D^{2} \Theta}{D t^{2}} \in L^{2}\left(0, T ; L^{2}(\Omega)\right)$,

3. $\frac{D^{3} \Theta}{D t^{3}} \in L^{2}\left(0, T ; L^{2}(\Omega)\right)$.

ASSUMPTION 3. The functions $\nu$, $\boldsymbol{f}$ and $g$ are assumed to verify:

1. $\nu$ is Lipschitz continuous and there exist two strictly positive constants $\nu_{1}$ and $\nu_{2}$ such that

$$
\nu_{1} \leq \nu(\xi) \leq \nu_{2}, \quad \forall \xi \in \mathbb{R}
$$

2. $f$ is Lipschitz with respect to its variable $\Theta$, i.e.,

$$
\|\boldsymbol{f}(\Theta)\|_{L^{\infty}\left(0, T ; L^{2}(\Omega)^{d}\right)} \leq K_{\boldsymbol{f}}\|\Theta\|_{L^{\infty}\left(0, T ; L^{2}(\Omega)^{d}\right)},
$$

where $K_{f}$ is a positive constant.

3. $g \in L^{2}\left(0, T ; L^{2}(\Omega)\right)$.

Following $[10,18]$, we consider the following necessary hypotheses on the velocity $\boldsymbol{u}$ and pressure $p$ : ASSUMPTION 4. The spaces $\mathbf{S}_{h}$ have the following approximation property: given a function $\boldsymbol{u} \in$ $H^{k+1}(\Omega)^{d}, k=1,2$, there exists $u_{h} \in \mathbf{S}_{h}$ such that

$$
\left\|\boldsymbol{u}-\boldsymbol{u}_{h}\right\|_{L^{2}(\Omega)^{d}}+h\left|\boldsymbol{u}-\boldsymbol{u}_{h}\right|_{H^{1}(\Omega)^{d}} \leq K h^{k+1}|\boldsymbol{u}|_{H^{k+1}(\Omega)^{d}}
$$

where $K$ is a positive constant independent of $h$.

ASSUMPTION 5. We assume that for all $p \in H^{1}(\Omega)$, there exists a function $p_{h} \in Q_{h}$ such that

$$
\left\|p-p_{h}\right\|_{L^{2}(\Omega)} \leq K h|p|_{H^{1}(\Omega)}
$$

where $K$ is a positive constant independent of $h$.

We introduce the projection operator $\Pi_{k-1}: L^{2}(\Omega) \longrightarrow[P]_{k-1}$ as defined in [10]:

$$
\Pi_{k-1}(p)=\arg \min \frac{1}{2} \int_{\Omega}\left(\Pi_{k-1} q-p\right)^{2} d \Omega, \quad \forall q \in[P]_{k-1},
$$

where $[P]_{k-1}$ is the discontinuous polynomial space defined as

$$
[P]_{k-1}=\left\{q \in L^{2}(\Omega):\left.\quad q\right|_{\mathcal{T}_{j}} \in P_{k-1}\left(\mathcal{T}_{j}\right), \quad \forall \mathcal{T}_{j} \in \Omega_{h}\right\} .
$$

Then, we have the following assumption on the projection operator defined in (7): 
ASSUMPTION 6. For all $p \in L^{2}(\Omega)$, the operator $\Pi_{k-1}$ defined in (7) is assumed to satisfy:

1. $\Pi_{k-1}: L^{2}(\Omega) \longrightarrow L^{2}(\Omega)$ is continuous and

$$
\left\|\Pi_{k-1} p\right\|_{L^{2}(\Omega)} \leq K\|p\|_{L^{2}(\Omega)}
$$

where $K$ is a positive constant independent of $h$.

2. The properties of $\Pi_{k-1}$ must be augmented by the approximation

$$
\left\|p-\Pi_{k-1} p\right\|_{L^{2}(\Omega)} \leq K^{\prime} h|p|_{H^{1}(\Omega)},
$$

where $K^{\prime}$ is a positive constant independent of $h$.

Finally, we introduce the operators required for the error analysis of the temperature solution. Hence, assuming that the subset $V_{h}$ is composed of polynomials of degree $k \geq 2$ on each element $\mathcal{T}_{j}, j=$ $1, \ldots, N_{e}$ of the partition $\Omega_{h}$. We define the following operators:

- The orthogonal projection operator $P_{0}: H^{-1}(\Omega) \longrightarrow V_{h}$

$$
\left(P_{0} \Theta, \phi\right)=(\Theta, \phi), \quad \forall \phi \in V_{h} .
$$

- The polynomial interpolant of degree $m$ for continuous functions $\Theta \in V_{h}, I_{m}: C^{0}(\bar{\Omega}) \longrightarrow V_{h}$

$$
I_{m} \Theta(\boldsymbol{x})=\sum_{j=1}^{M} \Theta\left(\boldsymbol{x}_{j}\right) \phi_{j}(\boldsymbol{x}), \quad \forall \boldsymbol{x} \in \bar{\Omega},
$$

where $\boldsymbol{x}_{j}(1 \leq j \leq M)$ are mesh points in the partition $\Omega_{h}$. Then, by the approximation theory, we have

$$
\left\|\Theta-I_{m} \Theta\right\|_{L^{2}(\Omega)} \leq K h^{m+1}|w|_{m+1} .
$$

- The linear continuous operator $O: H_{0}^{1}(\Omega) \longrightarrow H^{-1}(\Omega)$

$$
\langle O \Theta, r\rangle=(\nabla \Theta, \nabla r), \quad \forall r \in H_{0}^{1}(\Omega),
$$

where $\langle\cdot, \cdot\rangle$ denotes the duality pairing. It is evident that $A$ is a symmetric positive definite operator on $H_{0}^{1}(\Omega)$.

- The discrete operator $O_{h}: V_{h} \longrightarrow V_{h}$,

$$
\left(O_{h} \Theta_{h}, \phi\right)=\left\langle A \Theta_{h}, \phi\right\rangle, \quad \forall \phi \in V_{h} .
$$

$O_{h}$ is also a symmetric positive definite operator on $V_{h}$.

- The Ritz projection operator $R: V_{h} \longrightarrow V_{h}$,

$$
(\nabla \Theta, \nabla r)=\left\langle A \Theta_{h}, \phi\right\rangle, \quad \forall \phi \in V_{h} .
$$

- The discrete operator $G_{h}: V_{h} \longrightarrow V_{h}$,

$$
\left(g_{h}, \phi\right)=(g, \phi), \quad \forall \phi \in V_{h} .
$$

Note that it is easy to verify that

$$
O_{h} R=P_{0} A \quad \text { and } \quad G_{h}=P_{0} g .
$$

Note that the assumptions introduced above are necessary for the existence and uniqueness of the solution of problem (1), and also for the a priori error analysis. 


\section{Solution of the convection-diffusion-Darcy problem}

In this section, we formulate the unified Galerkin-characteristic finite element approximation used for solving the coupled convection-diffusion-Darcy problem (1). Next, we derive the second-order CrankNicolson scheme applied for the time integration and prove the existence and the uniqueness of the solution.

\subsection{Unified Galerkin-characteristic finite element approximation}

As in most finite element methods, we begin with the variational formulation that reads as: Find $(\boldsymbol{u}, p, \Theta)$ in $\mathbf{H}_{0}($ div,$\Omega) \times L_{0}^{2}(\Omega) \times H_{0}^{1}(\Omega)$ such that

$$
\begin{aligned}
\int_{\Omega} \nu(\Theta) \boldsymbol{u} \cdot \mathbf{s} d \Omega-\int_{\Omega} p \nabla \cdot \mathbf{s} d \Omega & =\int_{\Omega} \boldsymbol{f}(\Theta) \cdot \mathbf{s} d \Omega, & & \forall \mathbf{s} \in \mathbf{H}_{0}(d i v, \Omega), \\
\int_{\Omega} q \nabla \cdot \boldsymbol{u} d \Omega & =0, & & \forall q \in L_{0}^{2}(\Omega), \\
\int_{\Omega} \frac{D \Theta}{D t} r d \Omega+\kappa \int_{\Omega} \nabla \Theta \cdot \nabla r d \Omega & =\int_{\Omega} g r d \Omega, & & \forall r \in H_{0}^{1}(\Omega) .
\end{aligned}
$$

Notice that it is evident to prove that any triplet $(\boldsymbol{u}, p, \Theta)$ in $\mathbf{H}_{0}(\operatorname{div}, \Omega) \times L_{0}^{2}(\Omega) \times H_{0}^{1}(\Omega)$ solving the problem (1) in the sense of distributions in $\Omega$ is a solution of the variational problem (17), see [14, 42] for more details. It is also worth noting that the main advantage of the proposed method is that it does not require the use of mixed formulations such as those widely employed in the literature, see for instance $[2,41]$. Thus, the conforming finite element spaces that we use are polynomials $P_{k}$ of the same degree $k, k \leq 2$, for all the solutions defined on the element $\mathcal{T}_{j}$ in $\Omega_{h}$. To approximate the velocity and pressure solutions of the Darcy equations (17a), we use the equal-order finite element pair $\left(\mathbf{S}_{h}, Q_{h}\right)$ defined in (3). Next, to approximate the temperature solution of the convection-diffusion equation (17b) we use the finite element space $R_{h}$ defined in (4).

Note that we can rewrite equations (17a) as

$$
\begin{array}{rlrl}
\mathcal{A}\left(\boldsymbol{u}_{h}, \mathbf{s}_{h}\right)-\mathcal{B}\left(p_{h}, \mathbf{s}_{h}\right) & =\mathcal{L}_{\boldsymbol{f}}\left(\mathbf{s}_{h}\right), & & \forall \mathbf{s}_{h} \in \mathbf{S}_{h}, \\
\mathcal{B}\left(q_{h}, \boldsymbol{u}_{h}\right)=0, & \forall q_{h} \in Q_{h},
\end{array}
$$

where $\mathcal{A}, \mathcal{B}$ are the bilinear forms and $\mathcal{L}_{f}$ is the linear form defined as

$$
\mathcal{A}\left(\boldsymbol{u}_{h}, \mathbf{s}_{h}\right)=\int_{\Omega} \nu(\Theta) \boldsymbol{u}_{h} \cdot \mathbf{s}_{h} d \Omega, \quad \mathcal{B}\left(p_{h}, \mathbf{s}_{h}\right)=\int_{\Omega} p_{h} \nabla \cdot \mathbf{s}_{h} d \Omega, \quad \mathcal{L}_{\boldsymbol{f}}\left(\mathbf{s}_{h}\right)=\int_{\Omega} \boldsymbol{f}\left(\Theta_{h}\right) \cdot \mathbf{s}_{h} d \Omega .
$$

Note that stable and accurate solutions of the discrete problem (18) are obtained for discrete spaces $\mathbf{S}_{h}$ and $Q_{h}$ satisfying the discrete inf-sup condition [12]:

$$
\sup _{\substack{\mathbf{s}_{h} \in \mathbf{S}_{h} \\ \mathbf{s}_{h} \neq 0}} \frac{\mathcal{B}\left(p_{h}, \mathbf{s}_{h}\right)}{\left\|\mathbf{s}_{h}\right\|_{H^{1}(\Omega)^{d}}} \geq K\left\|p_{h}\right\|_{L^{2}(\Omega)}, \quad \forall p_{h} \in Q_{h},
$$


with $K>0$ being a constant independent of $h$. It is well known however that the pair $\left(\mathbf{S}_{h}, Q_{h}\right)$ does not verify the inf-sup condition associated with the mixed form (18), see $[10,11,30]$ for further details. Then, the discrete weak problem is not stable and a stabilization techniques is required. To stabilize (18) we use the polynomial pressure-projection stabilization method introduced in $[9,10]$, and thus the velocity-pressure space $\left(\mathbf{S}_{h}, Q_{h}\right)$ verifies a stabilized form of the inf-sup condition (19). We recall the following result whose proof can be found in [10].

Lemma 1. Let $\mathbf{S}_{h}$ and $Q_{h}$ be the spaces defined in (3). Then, there exist positive constants $K_{1}$ and $K_{2}$ whose values are independent of $h$ such that

$$
\sup _{\substack{\mathbf{s}_{h} \in \mathbf{S}_{h} \\ \mathbf{s}_{h} \neq 0}} \frac{\mathcal{B}\left(p_{h}, \mathbf{s}_{h}\right)}{\left\|\mathbf{s}_{h}\right\|_{H^{1}(\Omega)^{d}}} \geq K_{1}\left\|p_{h}\right\|_{L^{2}(\Omega)}-K_{2}\left\|p_{h}-\Pi_{k-1} p_{h}\right\|_{L^{2}(\Omega)}, \quad \forall p_{h} \in Q_{h},
$$

where $\Pi_{k-1}$ is the projection operator defined in (7).

Thereafter, the stabilized weak form of equations (17a) reads as: Find $\left(\boldsymbol{u}_{h}, p_{h}\right) \in \mathbf{S}_{h} \times Q_{h}$ such that

$$
\begin{aligned}
\mathcal{A}\left(\boldsymbol{u}_{h}, \mathbf{s}_{h}\right)-\mathcal{B}\left(p_{h}, \mathbf{s}_{h}\right) & =\mathcal{L}_{f}\left(\mathbf{s}_{h}\right), & & \forall \mathbf{s}_{h} \in \mathbf{S}_{h}, \\
\mathcal{B}\left(q_{h}, \boldsymbol{u}_{h}\right) & =\mathcal{D}\left(p_{h}, q_{h}\right), & & \forall q_{h} \in Q_{h},
\end{aligned}
$$

where $\mathcal{D}$ is the bilinear form defined as

$$
\mathcal{D}\left(p_{h}, q_{h}\right)=\int_{\Omega}\left(p_{h}-\Pi_{k-1} p_{h}\right)\left(q_{h}-\Pi_{k-1} q_{h}\right) d \Omega .
$$

Note that, in contrast to other stabilization techniques, the unified finite element method offers attractive computational properties as, it does not require calculation of higher order derivatives and always leads to symmetric linear systems. The stabilized unified finite element method has been investigated and analyzed for Stokes and Darcy problems in $[9,10,18]$. The obtained results have shown that the method is unconditionally stable and it allows to achieve optimal accuracy with respect to solution regularity.

Next, to solve the convection-diffusion equation (17b), we use the modified method of characteristics for the transport term. This class of methods has been used for solving many convection-dominated flow problems, see for example $[6,22,19,38,36,37,42]$. The main idea is to treat the transport part $\frac{D \Theta}{D t}$ of equation (17b) separately in the finite element discretization. Then, new temperature solution is approximated at each time subinterval $\left[t_{n}, t_{n+1}\right]$ using the characteristic curves associated with the material derivative (1c). We denote by $\boldsymbol{X}\left(\boldsymbol{x}, t_{n+1} ; t\right)$ the characteristic curves associated with the material derivative (1c). These are the solutions of the ordinary differential equations

$$
\begin{aligned}
\frac{d \boldsymbol{X}\left(\boldsymbol{x}, t_{n+1} ; t\right)}{d t} & =\boldsymbol{u}\left(\boldsymbol{X}\left(\boldsymbol{x}, t_{n+1} ; t\right), t\right), \quad \forall(t, \boldsymbol{x}) \in\left[t_{n}, t_{n+1}\right] \times \bar{\Omega}, \\
\boldsymbol{X}\left(\boldsymbol{x}, t_{n+1} ; t_{n+1}\right) & =\boldsymbol{x} .
\end{aligned}
$$

Notice that we assume that the velocity field $\boldsymbol{u}$ satisfies Assumption 1 which guarantees the existence and uniqueness of the solution of (22) for all times $t$, see for instance [28]. The solutions of equation 
(22) represent the departure points at time $t$ of a particle passing through the point $\boldsymbol{x}$ at time $t=t_{n+1}$. Then, the unique solution of (22) can be written for all $(t, \boldsymbol{x})$ in $\left[t_{n}, t_{n+1}\right] \times \bar{\Omega}$ as

$$
\boldsymbol{X}\left(\boldsymbol{x}, t_{n+1} ; t_{n}\right)=\boldsymbol{x}-\int_{t_{n}}^{t_{n+1}} \boldsymbol{u}\left(t, \boldsymbol{X}\left(\boldsymbol{x}, t_{n+1} ; t\right)\right) d t
$$

To obtain the departure points $\left\{\boldsymbol{X}_{h j}^{n}\right\}$ for each mesh point $\boldsymbol{x}_{j}, j=1, \ldots, M$, we use the algorithm proposed in [48] which accurately solves (22) with a second-order accuracy. We write the solution of (22) in the form of

$$
\boldsymbol{X}_{h j}^{n}=\boldsymbol{x}_{j}-\boldsymbol{d}_{h j}, \quad j=1, \ldots, M,
$$

where the displacement $\boldsymbol{d}_{h j}$ is calculated by the iterative procedure

$$
\begin{aligned}
\boldsymbol{d}_{h j}^{(0)} & =\frac{\Delta t}{2}\left(3 \boldsymbol{u}_{h}^{n}\left(\boldsymbol{x}_{j}\right)-\boldsymbol{u}_{h}^{n-1}\left(\boldsymbol{x}_{j}\right)\right) \\
\boldsymbol{d}_{h j}^{(k+1)} & =\frac{\Delta t}{2}\left(3 \boldsymbol{u}_{h}^{n}\left(\boldsymbol{x}_{j}-\frac{1}{2} d_{h j}^{(k)}\right)-\boldsymbol{u}_{h}^{n-1}\left(\boldsymbol{x}_{j}-\frac{1}{2} d_{h j}^{(k)}\right)\right), \quad k=0,1, \ldots
\end{aligned}
$$

To evaluate values of the approximate velocities $\boldsymbol{u}_{h}^{n}\left(\boldsymbol{x}_{j}-\frac{1}{2} \boldsymbol{d}_{h j}^{(k)}\right)$ and $\boldsymbol{u}_{h}^{n-1}\left(\boldsymbol{x}_{j}-\frac{1}{2} \boldsymbol{d}_{h j}^{(k)}\right)$ in (25), we first identify the mesh element $\widehat{\mathcal{T}}_{j}$ where $\boldsymbol{x}_{j}-\frac{1}{2} \boldsymbol{d}_{h j}^{(k)}$ resides. Then, a finite element interpolation on $\widehat{\mathcal{T}}_{j}$ is performed according to (2). In the numerical simulations obtained, the iterations in (25) were continued until the trajectory changed by less than $10^{-7}$. However, in practice it is not recommended to repeat the iteration process more than a few times due to efficiency considerations.

Now, we assume that the pairs $\left(\boldsymbol{X}_{h j}^{n}, \widehat{\mathcal{T}}_{j}\right)$ along with the mesh point values $\left\{\mathcal{C}_{j}^{n}\right\}$ are known for all $j=1, \ldots, M$, we can compute the values $\left\{\widehat{\mathcal{C}}_{j}^{n}\right\}$ as

$$
\widehat{\mathcal{C}}_{j}^{n}:=\Theta_{h}^{n}\left(\boldsymbol{X}_{h j}^{n}\right)=\sum_{k=1}^{M} \mathcal{C}_{k} \phi\left(\boldsymbol{X}_{h j}^{n}\right) .
$$

Then, the solution $\left\{\widehat{\Theta}_{h}^{n}\right\}$ of the convection-diffusion equation (1b) is obtained by

$$
\widehat{\Theta}_{h}^{n}(\boldsymbol{x})=\sum_{j=1}^{M} \widehat{\mathcal{C}}_{j}^{n} \phi_{j}(\boldsymbol{x}) .
$$

Note that (26) and (27) are respectively, the local and global approximations of the solution $c_{h}^{n}$ at the departure points $\boldsymbol{X}_{h j}^{n}$.

\subsection{Time integration procedure}

To solve the reaction-diffusion terms in (1b) we use the Crank-Nicolson scheme for the time integration as

$$
\frac{\Theta_{h}^{n+1}-\widehat{\Theta}_{h}^{n}}{\Delta t}-\frac{\kappa}{2} \nabla^{2} \Theta_{h}^{n+1}=g_{h}^{n}+\frac{\kappa}{2} \nabla^{2} \widehat{\Theta}_{h}^{n}
$$


Then, we obtain the discretization of the convection diffusion problem (17) as:

Find $\left(\boldsymbol{u}_{h}^{n+1}, p_{h}^{n+1}, \Theta_{h}^{n+1}\right)$ in $\mathbf{S}_{h} \times Q_{h} \times R_{h}$ such that

$$
\begin{gathered}
\int_{\Omega} \nu\left(\widehat{\Theta}_{h}^{n}\right) \boldsymbol{u}_{h}^{n+1} \cdot \mathbf{s}_{h} d \Omega-\int_{\Omega} p_{h}^{n+1} \nabla \cdot \mathbf{s}_{h} d \Omega=\int_{\Omega} \boldsymbol{f}\left(\widehat{\Theta}_{h}^{n}\right) \cdot \mathbf{s}_{h} d \Omega, \quad \forall \mathbf{s}_{h} \in \mathbf{S}_{h}, \\
\int_{\Omega} q_{h} \nabla \cdot \boldsymbol{u}_{h}^{n+1} d \Omega=\int_{\Omega}\left(p_{h}^{n+1}-\Pi_{k-1} p_{h}^{n+1}\right)\left(q_{h}-\Pi_{k-1} q_{h}\right) d \Omega, \quad \forall q_{h} \in Q_{h}, \\
\int_{\Omega} \frac{\Theta_{h}^{n+1}-\widehat{\Theta}_{h}^{n}}{\Delta t} r_{h} d \Omega+\frac{\kappa}{2} \int_{\Omega} \nabla \Theta_{h}^{n+1} \cdot \nabla r_{h} d \Omega=\int_{\Omega} g_{h}^{n} r_{h} d \Omega+ \\
\frac{\kappa}{2} \int_{\Omega} \nabla \widehat{\Theta}_{h}^{n} \cdot \nabla r_{h} d \Omega, \quad \forall r \in R_{h},
\end{gathered}
$$

where $\widehat{\Theta}_{h}^{n}$ are the characteristics curves obtained by (27) and $g_{h}^{n}$ the function given by

$$
g_{h}^{n}=\frac{1}{\Delta t} \int_{t_{n}}^{t_{n+1}} g_{h}(s) d s .
$$

It is worth mentioning that by using the proposed Galerkin-characteristic approach, the time derivative and the convection terms are combined as a directional derivative along the particles trajectories, leading to a characteristic time-stepping procedure. This results in a substantial reduction in both the computational cost and the time truncation errors in the diffusion-reaction part of the problem under study. Needless to mention that most of conventional Eulerian-based Galerkin finite element methods are subject to stability conditions which impose strict limitations on the time steps considered in the numerical simulations, see for instance [32, 34, 47]. For the existence and uniqueness of the solution of (29), we have the following Theorem:

THEOREM 1. At each time step $t_{n}$ and for given $\widehat{\Theta}_{h}^{n} \in R_{h}$, problem (29) has a unique solution $\left(\boldsymbol{u}_{h}^{n+1}, p_{h}^{n+1}, \Theta_{h}^{n+1}\right)$ in $\mathbf{S}_{h} \times Q_{h} \times R_{h}$ that verifies the following bounds:

$$
\begin{aligned}
& \left\|\boldsymbol{u}_{h}^{n+1}\right\|_{L^{2}(\Omega)^{d}}^{2} \leq\left(\frac{K_{\boldsymbol{f}}}{\nu_{1}}\right)^{2}\left\|\widehat{\Theta}_{h}^{n}\right\|_{L^{2}(\Omega)^{d}}^{2}+K h^{2}\left|p_{h}^{n+1}\right|_{H^{1}(\Omega)}^{2}, \\
& \left\|\Theta_{h}^{n+1}\right\|_{L^{2}(\Omega)}-\left\|\widehat{\Theta}_{h}^{n}\right\|_{L^{2}(\Omega)} \leq \Delta t\left\|g_{h}^{n}\right\|_{L^{2}(\Omega)},
\end{aligned}
$$

where $K$ is a positive constant independent of $h$.

PROOF. It is clear that the Darcy equations (21) have a unique solution since they satisfy the inf-sup condition (20). Let $a$ and $b$ be two real numbers. For any positive real number $\epsilon$, we have the well-known Young's inequality

$$
a b \leq \frac{1}{2 \epsilon} a^{2}+\frac{1}{2} \epsilon b^{2} .
$$


By testing equations (29a) with $\mathbf{s}_{h}=\boldsymbol{u}_{h}$ and $q_{h}=p_{h}$ and using the Cauchy-Schwarz inequality and (32) with $\epsilon=\frac{\nu_{1}}{K_{f}}$, along with Assumptions 3 and 6, we immediately derive (30).

Next, knowing $\boldsymbol{u}_{h}^{n} \in \mathbf{S}_{h}$ and thus $\widehat{\Theta}_{h}^{n}$, the convection-diffusion equation (29b) admits also a unique solution $\Theta_{h}^{n+1} \in R_{h}$. Therefore, if we take $r_{h}=\Theta_{h}^{n+1}+\widehat{\Theta}_{h}^{n}$ in (29b), we obtain and use the CauchySchwarz and triangle inequalities, we easily get the inequality (31).

\section{A priori error analysis}

In this section, we present the a priori error estimates in the $L^{2}$-norm corresponding to the velocity, pressure temperature solutions.

\subsection{Error estimates for velocity and pressure}

Let $(\boldsymbol{u}, p)$ and $\left(\boldsymbol{u}_{h}^{n}, p_{h}^{n}\right)$ be the solutions of the Darcy problems (17a) and (21), respectively. Equations (17a) can be written in compact form as

$$
\mathcal{S}\left(\boldsymbol{u}^{n}, p^{n} ; \mathbf{s}, q\right)=\mathcal{L}_{\boldsymbol{f}}(\mathbf{s}), \quad \forall(\mathbf{s}, q) \in H_{0}^{1}(\Omega) \times L_{0}^{2}(\Omega),
$$

with $\mathcal{S}$ being the bilinear form given by

$$
\mathcal{S}\left(\boldsymbol{u}^{n}, p^{n} ; \mathbf{s}, q\right)=\mathcal{A}\left(\boldsymbol{u}^{n}, \mathbf{s}\right)-\mathcal{B}\left(p^{n}, \mathbf{s}\right)+\mathcal{B}\left(q, \boldsymbol{u}^{n}\right) .
$$

Similarly, we can write (21) in the following form

$$
\widetilde{\mathcal{S}}\left(\boldsymbol{u}_{h}^{n}, p_{h}^{n} ; \mathbf{s}_{h}, q_{h}\right)=\mathcal{L}_{\boldsymbol{f}}\left(\mathbf{s}_{h}\right), \quad \forall\left(\mathbf{s}_{h}, q_{h}\right) \in \mathbf{S}_{h} \times Q_{h},
$$

where $\widetilde{\mathcal{S}}$ is the bilinear form given by

$$
\widetilde{\mathcal{S}}\left(\boldsymbol{u}_{h}^{n}, p_{h}^{n} ; \mathbf{s}_{h}, q_{h}\right)=\mathcal{A}\left(\boldsymbol{u}_{h}^{n}, \mathbf{s}_{h}\right)-\mathcal{B}\left(p_{h}^{n}, \mathbf{s}_{h}\right)+\mathcal{B}\left(q_{h}, \boldsymbol{u}_{h}^{n}\right)-\mathcal{D}\left(p_{h}^{n}, q_{h}\right) .
$$

The stability of the variational problem (21) is provided by the following Theorem associated to the weak coercivity bound, the proof for which can be found in [10]:

THEOREM 2. Let $\left(\mathbf{S}_{h}, Q_{h}\right)$ be the pair of spaces defined in (3). Then, there exists a positive constant $K$ whose value is independent of huch that

$$
\sup _{\left(\mathbf{s}_{h}, q_{h}\right) \in \mathbf{S}_{h} \times Q_{h}} \frac{\widetilde{\mathcal{S}}\left(\boldsymbol{u}_{h}^{n}, p_{h}^{n} ; \mathbf{s}_{h}, q_{h}\right)}{\left|\mathbf{s}_{h}\right|_{H^{1}(\Omega)^{d}}+\left\|q_{h}\right\|_{L^{2}(\Omega)}} \geq K\left(\left|\boldsymbol{u}_{h}\right|_{H^{1}(\Omega)^{d}}+\left\|p_{h}\right\|_{L^{2}(\Omega)}\right), \quad \forall\left(\boldsymbol{u}_{h}, p_{h}\right) \in \mathbf{S}_{h} \times Q_{h} .
$$

Next, to establish a priori error estimates for the stabilized solutions of (34), we shall use the following Theorem, , the proof for which can be found in [42]:

THEOREM 3. Let $\left(\mathbf{S}_{h}, Q_{h}\right)$ be the pair of spaces defined in (3), let $(\boldsymbol{u}, p) \in \mathbf{H}_{0}($ div,$\Omega) \times L_{0}^{2}(\Omega)$ be the solution of the Darcy problem (17a) and let $\left(\boldsymbol{u}_{h}, p_{h}\right) \in \mathbf{S}_{h} \times Q_{h}$ be the solution of the stabilized mixed problem (21), where the operator $\Pi_{k-1}$ defined in (7) satisfies (6). Then, there exists a positive constant $K$ whose value is independent of $h$ such that

$$
\left|\boldsymbol{u}^{n}-\boldsymbol{u}_{h}^{n}\right|_{H^{1}(\Omega)^{d}}+\left\|p^{n}-p_{h}^{n}\right\|_{L^{2}(\Omega)} \leq K\left(\inf _{\boldsymbol{w}_{h} \in \mathbf{S}_{h}}\left|\boldsymbol{u}^{n}-\boldsymbol{w}_{h}^{n}\right|_{H^{1}(\Omega)^{d}}+\inf _{l_{h} \in Q_{h}}\left\|p^{n}-l_{h}^{n}\right\|_{L^{2}(\Omega)}+\right.
$$




$$
\left.\left\|p^{n}-\Pi_{k-1} p^{n}\right\|_{L^{2}(\Omega)}+\left\|\widehat{\Theta}_{h}^{n-1}-\Theta^{n-1}\right\|_{L^{2}(\Omega)^{d}}\right) .
$$

Notice that Theorem 3 shows that solutions of (34) converge optimally in regard to the solution regularity. Thus, we deduce, from Theorem 3, the following result for the error estimates of the velocity and pressure solutions:

Lemma 2. Let $(\boldsymbol{u}, p)$ be the solution of (17a) and $\left(\boldsymbol{u}_{h}^{n}, p_{h}^{n}\right)$ be the solution of (21) at each time step $t_{n}$. Under Assumptions 4, 5 and 6, there exists a positive constant $K$ depending on $\boldsymbol{u}$ and $p$ such that

$$
\left|\boldsymbol{u}^{n}-\boldsymbol{u}_{h}^{n}\right|_{H^{1}(\Omega)^{d}}+\left\|p^{n}-p_{h}^{n}\right\|_{L^{2}(\Omega)} \leq K\left(h\left|\boldsymbol{u}^{n}\right|_{H^{2}(\Omega)^{d}}+h\left|p^{n}\right|_{H^{1}(\Omega)^{d}}+\left\|\widehat{\Theta}_{h}^{n-1}-\Theta^{n-1}\right\|_{L^{2}(\Omega)^{d}}\right) .
$$

Proof. Under Assumptions 4, 5 and 6, and by taking into account the inequality (36) of Theorem 3, we immediately obtain (37).

\subsection{Error estimates for the temperature}

To establish error estimates for the temperature solution, we consider the one-step method for the computation of the approximate solutions $\left\{\boldsymbol{X}_{h}^{n}\right\}$ of (22) which has the following form [6, 20, 42]:

$$
\boldsymbol{X}_{h}^{n}(\boldsymbol{x})=\boldsymbol{x}-\Delta t \Phi_{\boldsymbol{u}_{h}}\left(t_{n+1}, \boldsymbol{x}, \Delta t\right), \quad \forall \boldsymbol{x} \in \Omega_{h},
$$

with $\Phi_{\boldsymbol{u}_{h}}\left(t_{n+1}, \boldsymbol{x}, \Delta t\right)$ being the incremental function. We also announce the following assumption that provides the convergence of $\boldsymbol{X}_{h}^{n}(\boldsymbol{x})$ to $\boldsymbol{X}_{h}\left(\boldsymbol{x}, t_{n+1} ; t_{n}\right)$ :

ASSUMPTION 7. We impose the following assumption:

1. There exists a real constant $0<\Delta t_{0}<1$ such that:

$$
\Phi_{\boldsymbol{u}_{h}}:[0, T] \times \Omega_{h} \times\left(0, \Delta t_{0}\right) \longrightarrow \mathbb{R}^{d},
$$

is a continuous function that only depends on $\boldsymbol{u}_{h}$.

2. For any $t \in[0, T]$ and $\boldsymbol{x} \in \Omega_{h}, \Phi_{\boldsymbol{u}_{h}}(t, \boldsymbol{x}, \Delta t) \longrightarrow \boldsymbol{u}_{h}(\boldsymbol{x}, t)$ as $\Delta t \longrightarrow 0$.

3. For any $t \in[0, T], \boldsymbol{x}, \mathbf{y} \in \Omega_{h}$ and $\Delta t \in\left(0, \Delta t_{0}\right)$, there exists a positive constant $K$ such that

$$
\left|\Phi_{\boldsymbol{u}_{h}}(t, \boldsymbol{x}, \Delta t)-\Phi_{\boldsymbol{u}_{h}}(t, \mathbf{y}, \Delta t)\right| \leq K|\boldsymbol{x}-\mathbf{y}| .
$$

4. There exists $\Delta t^{*}$, with $0<\Delta t^{*}<\Delta t_{0}$, such that for $\Delta t \in\left(0, \Delta t^{*}\right)$ and $h \in\left(0, h_{0}\right)$, the method is absolutely stable.

5. The method is of order $p$, where $p$ is an integer larger than 0. This means that, if

$$
\boldsymbol{X}_{h}\left(\boldsymbol{x}, t_{n+1} ; t_{n}\right)=\boldsymbol{x}-\int_{t_{n}}^{t_{n+1}} \boldsymbol{u}_{h}\left(t, \boldsymbol{X}_{h}\left(\boldsymbol{x}, t_{n+1} ; t\right)\right) d t
$$

is the exact solution of (22) for any $\boldsymbol{x} \in \Omega_{h}$, and we assume that $\boldsymbol{u}_{h}(\boldsymbol{x}, t)$ is sufficiently smooth in time, then for all $\Delta t \in\left(0, \Delta t^{*}\right), h \in\left(0, h_{0}\right)$ and $t_{n} \in(0, T]$, we have

$$
\left|\boldsymbol{X}_{h}\left(\boldsymbol{x}, t_{n+1} ; t_{n}\right)-\boldsymbol{X}_{h}^{n}(\boldsymbol{x})\right|=\mathcal{O}\left(\Delta t^{p+1}\right) .
$$


We also recall the following result concerning convergence of the characteristics, the proof for which can be found in $[6,20]$ :

Lemma 3. Assume that for each time subinterval $\left[t_{n}, t_{n+1}\right]$, the points $\left\{\boldsymbol{X}_{h}^{n}(\boldsymbol{x})\right\}$ are calculated by the one-step method (38) such that Assumption 7 holds. Then

$$
\left\|\boldsymbol{X}\left(\boldsymbol{x}, t_{n+1} ; t_{n}\right)-\boldsymbol{X}_{h}^{n}(\boldsymbol{x})\right\|_{L^{\infty}\left(0, T ; L^{2}(\Omega)\right)} \leq K \Delta t\left\|\boldsymbol{u}-\boldsymbol{u}_{h}\right\|_{L^{\infty}\left(0, T ; L^{2}(\Omega)\right)}+\mathcal{O}\left(\Delta t^{p+1}\right),
$$

where $K$ is a positive constant defined by $K=e^{\Delta t^{*}|\nabla \boldsymbol{u}|_{L \infty(0, T, \Omega)}}$.

Notice that it is necessary to impose a condition on $\Delta t$ to approximate the departure points $\left\{\boldsymbol{X}_{h}^{n}(\boldsymbol{x})\right\}$, under which the functional iterative $\boldsymbol{d}_{h j}$ defined in (24) converges.

Lemma 4. Suppose that Assumption 2 holds and that for all $n$

$$
\Delta t\left\|\nabla \Theta_{h}^{n}\right\|_{L^{\infty}(\Omega)}<2
$$

Then, (39) represents an estimate of the committed error to compute the departure points $\left\{\boldsymbol{X}_{h}^{n}(\boldsymbol{x})\right\}$.

As a consequence, we shall study stability and convergence for the temperature solution in the $L^{2}$ norm. To this end, we recall the following result of reference [6] (Lemma 6 in page 40) concerning the stability of the Galerkin-characteristic step:

Lemma 5. Assume that Assumption 7 holds, then for any $t_{n} \in[0, T]$, we have

$$
\left\|\widehat{\Theta}_{h}^{n}\right\|_{L^{2}(\Omega)} \leq\left\|\Theta_{h}^{n}\right\|_{L^{2}(\Omega)}
$$

Next, to prove convergence of stabilized solutions of (29b), we shall use the following Theorem:

THEOREM 4. Assume that Assumption 7 holds, then for any $t_{n} \in[0, T]$ we have

$$
\left\|\Theta_{h}^{n+1}\right\|_{L^{2}(\Omega)}-\left\|\Theta_{h}^{n}\right\|_{L^{2}(\Omega)} \leq \Delta t\left\|g_{h}^{n}\right\|_{L^{2}(\Omega)}
$$

PROOF. If we take $r_{h}=\Theta_{h}^{n+1}+\widehat{\Theta}_{h}^{n}$ in $(29 \mathrm{~b})$, we obtain

$$
\left\|\Theta_{h}^{n+1}\right\|^{2}-\left\|\widehat{\Theta}_{h}^{n}\right\|^{2}+\leq\left(\Delta t g_{h}^{n}, \Theta_{h}^{n+1}+\widehat{\Theta}_{h}^{n}\right)
$$

Then, using Cauchy-Schwarz and triangle inequalities together with (41), we immediately obtain (42) for each time step $t_{n}$.

Next, referring to [33] and under an additional assumption that the approximate solutions $\boldsymbol{u}_{h}$ are exponentially stable, it follows that

$$
\left\|\boldsymbol{u}-\boldsymbol{u}_{h}\right\| \leq \beta(t) h^{m+1} \quad \forall t \in[0, T]
$$

where the function $\beta(t)$ is an error constant independent of $h$ given by

$$
\beta(t)=\beta_{0} e^{\beta_{0} t} \tau^{\frac{1-m}{2}}(t)
$$


with $\tau(t)=\min (t, 1)$ and $\beta_{0}$ being a constant that depends on the solution $\boldsymbol{u}$. If $h$ is sufficiently small then, the approximation (43) holds on any interval of time over which the Dirichlet norm of $\boldsymbol{u}$ is bounded i.e., there exists a constant $K_{\beta}>0$ such that

$$
\sup _{[0, T)}\|\nabla \boldsymbol{u}\| \leq K_{\beta} .
$$

For the temperature solution, we consider the standard estimates of $\Theta-R \Theta$ and $\Theta-P_{0} \Theta$ which are presented in the following Lemma [7], where $R$ and $P_{0}$ are the Ritz and orthogonal projection operators respectively defined in Section 3:

LEMMA 1. If $\Theta$ belongs to $L^{\infty}\left(0, T ; H^{r}(\Omega)\right)$. Then, for all $t \in[0, T]$, there exist positive constants $K_{1}$ and $K_{2}$ such that for $1 \leq r \leq m+1$ the solution $\Theta$ of the transport-diffusion equation (1b) satisfies

$$
\|\Theta-R \Theta\|_{L^{\infty}\left(0, T ; L^{2}(\Omega)\right)}+h\|\Theta-R \Theta\|_{L^{\infty}\left(0, T ; H^{1}(\Omega)\right)} \leq K_{1} h^{r}\|\Theta\|_{L^{\infty}\left(0, T ; H^{r}(\Omega)\right)},
$$

and

$$
\left\|\Theta-P_{0} \Theta\right\|_{L^{\infty}\left(0, T ; L^{2}(\Omega)\right)}+h\left\|\Theta-P_{0} \Theta\right\|_{L^{\infty}\left(0, T ; H^{1}(\Omega)\right)} \leq K_{2} h^{r}\|\Theta\|_{L^{\infty}\left(0, T ; H^{r}(\Omega)\right)} .
$$

We also recall the following auxiliary results concerning properties of the mapping $\boldsymbol{x} \longrightarrow \boldsymbol{X}(\boldsymbol{x}, s, t)$ and $\boldsymbol{x} \longrightarrow \boldsymbol{X}_{h}(\boldsymbol{x}, s, t)$ whose proofs can be found in [46].

LemMa 2. Suppose that Assumption 7 holds, then the mapping $\boldsymbol{x} \longrightarrow \boldsymbol{X}_{h}(\boldsymbol{x}, s ; t)$ is a quasi-isometric homeomorphism of $\Omega$ into itself with an a.e zero Jacobian determinant.

Another interesting result related with the homeomorphisms of the previous Lemma is presented in the following Lemma [35]:

LEMMA 3. Let $\boldsymbol{X}(\boldsymbol{x}, s ; t)$ be the unique solution of (22) and assume that a quasi-isometric homeomorphism $\boldsymbol{x} \longrightarrow \boldsymbol{X}(\boldsymbol{x}, s ; t)$ is of class $\Theta^{r-1,1}(\bar{\Omega}), r \geq 1$. Let $f \in W^{r, p}(\bar{\Omega})$ and $h=f(\boldsymbol{X}(\boldsymbol{x}, s ; t))$, then $h \in \mathbf{W}^{r, p}(\bar{\Omega})$ and there exist positive constants $K_{1}$ and $K_{2}$ such that

$$
K_{1}\|f\|_{r, p} \leq\|h\|_{r, p} \leq K_{2}\|f\|_{r, p}
$$

Thus, since $\boldsymbol{x} \longrightarrow \boldsymbol{X}(\boldsymbol{x}, s ; t)$ defines a quasi-isometric homeomorphism of $\Omega$ onto itself, at each time $\delta \in\left[t_{n}, t_{n+1}\right]$ we can introduce the ephemeral Ritz projection and $L^{2}$-projection operators $R \widehat{\Theta}$ and $P_{0} \widehat{\Theta}$ respectively, as follows:

$$
\begin{aligned}
R \widehat{\Theta}(\boldsymbol{x}, \delta) & =z(\boldsymbol{x}, \delta)=\sum_{j=1}^{M} z_{j}(\delta) \phi_{j}(\boldsymbol{x}), \\
P_{0} \widehat{\Theta}(\boldsymbol{x}, \delta) & =\sum_{j=1}^{M} P_{0} \widehat{\Theta}_{j}(\delta) \phi_{j}(\boldsymbol{x}),
\end{aligned}
$$

such that $\forall \phi_{h} \in V_{h}$

$$
\begin{aligned}
\left(K \nabla z(\boldsymbol{x}, \delta), \nabla \phi_{h}\right) & =\left(K \nabla \widehat{\Theta}(\boldsymbol{x}, \delta), \nabla \phi_{h}\right)=P_{0}\left(g\left(\boldsymbol{X}^{\delta}, \delta\right)-\frac{D \Theta\left(\boldsymbol{X}^{\delta}, \delta\right)}{D \delta}\right) \\
\left(P_{0} \widehat{\Theta}(\boldsymbol{x}, \delta), \phi_{h}\right) & =\left(\widehat{\Theta}(\boldsymbol{x}, \delta), \phi_{h}\right),
\end{aligned}
$$


where $\widehat{\Theta}(\boldsymbol{x}, \delta)=\Theta\left(\boldsymbol{X}^{\delta}, \delta\right)$ with $\boldsymbol{X}^{\delta}=\boldsymbol{X}\left(\boldsymbol{x}, t_{n+1} ; \delta\right)$. Note that according to the definitions of $O_{h}, P_{0}$ and equation (48) it follows that

$$
O_{h} z=P_{0}\left(g\left(\boldsymbol{X}^{\delta}, \delta\right)-\frac{D \Theta\left(\boldsymbol{X}^{\delta}, \delta\right)}{D \delta}\right) .
$$

Consequently, for $t=t_{n+1}, \quad z^{n+1}=R \Theta^{n+1}$ and $\Theta^{n+1}=P_{0} \Theta^{n+1}$ for all $n$, and by virtue of Lemma 1 and Lemma 3, we have the following result [6]:

Lemma 4. Assume that $\Theta \in L^{\infty}\left(0, T ; H^{m+1}\right)$. Then, for all $\delta \in\left[t_{n}, t_{n+1}\right]$ there exist positive constants $K_{1}$ and $K_{2}$ such that

$$
\|\widehat{\Theta}-z\|_{L^{2}(\Omega)}+h\|\widehat{\Theta}-z\|_{H^{1}(\Omega)} \leq K_{1} h^{m+1}\|\Theta\|_{H^{m+1}(\Omega)},
$$

and

$$
\left\|\widehat{\Theta}-P_{0} \widehat{\Theta}\right\| \leq K_{2} h^{m+1}\|\Theta\|_{H^{m+1}(\Omega)} .
$$

Next, by combining (1b) and (49), we obtain

$$
\frac{\partial z}{\partial \delta}+O_{h} z=P_{0}\left(g\left(\boldsymbol{X}^{\delta}, \delta\right)+\frac{\partial z}{\partial \delta}-\frac{D \Theta\left(\boldsymbol{X}^{\delta}, \delta\right)}{D \delta}\right) .
$$

Note that each term of (50) is an element of $V_{h}$. Now, we discretize (50) by Crank-Nicolson scheme as follows

$$
\begin{aligned}
z^{n+1} & =E_{h} z^{n}+\frac{\Delta t}{2} S_{h}\left(P_{0} g^{n+1}+P_{0} \widehat{g}^{n}\right)+S_{h} P_{0}\left(z^{n+1}-z^{n}\right)-\Delta t S_{h} P_{0}\left(\frac{D \Theta\left(\boldsymbol{X}^{\delta^{\prime}}, \delta^{\prime}\right)}{D \delta}\right) \\
& \equiv \sum_{i=1}^{4} \mathcal{E}_{i}
\end{aligned}
$$

where $\delta^{\prime}=t_{n}+\frac{\Delta t}{2}$ and the operators $E_{h}: V_{h} \longrightarrow V_{h}$ and $S_{h}: V_{h} \longrightarrow V_{h}$ are given by

$$
E_{h} v_{h}=\left(\frac{I_{h}-\frac{\Delta t}{2} O_{h}}{I_{h}+\frac{\Delta t}{2} O_{h}}\right) v_{h}, \quad S_{h} v_{h}=\left(\frac{I_{h}}{I_{h}+\frac{\Delta t}{2} O_{h}}\right) v_{h},
$$

where $I_{h}: V_{h} \longrightarrow V_{h}$ is the identity operator and $E_{h}$ and $S_{h}$ verify $\left\|\left|E_{h} \|\right|<1\right.$ and $\|\left|S_{h} \|\right|<1$, with $\left\||\cdot \||\right.$ being the operator norm. Hence, we have the following result for the error estimate in the $L^{2}$-norm corresponding to the temperature solution:

THEOREM 5. Assume that the following hypotheses hold

1. $h=\mathcal{O}(\Delta t)$,

2. Assumption 2,

3. Assumption 7 ,

4. The time step $\Delta t$ satisfies the condition (40). 
Then, there exists a positive constant $K$ such that

$$
\begin{aligned}
\max _{0 \leq t_{n} \leq T}\left\|\Theta\left(t_{n}\right)-\Theta_{h}^{n}\right\| \leq & K h^{m+1}\|\Theta\|_{L^{\infty}\left(0, T ; H^{m+1}(\Omega)\right)}+K\left(\frac{h^{m+1}}{\Delta t}+\max _{t \in(0, T)} \beta(t) h^{m+1}+\right. \\
& \left.\mathcal{O}\left(\Delta t^{p}\right)\right)\|\Theta\|_{L^{\infty}\left(0, T ; W^{m+1, \infty}(\Omega)\right)}+K h^{m+1}\left\|\Theta_{t}\right\|_{L^{2}\left(0, T ; H^{m+1}(\Omega)\right)}+ \\
& K \Delta t^{2}\left\|\frac{D^{3} \Theta}{D t^{3}}\right\|_{L^{2}\left(0, T ; L^{2}(\Omega)\right)},
\end{aligned}
$$

where $\beta(t)$ is the error constant defined in (44) that depends on the solution $\boldsymbol{u}$.

Proof. We set

$$
\begin{aligned}
\Theta^{n+1}-\Theta_{h}^{n+1} & =\left(\Theta^{n+1}-z^{n+1}\right)+\left(z^{n+1}-\Theta_{h}^{n+1}\right), \\
& \equiv \rho^{n+1}+\xi^{n+1} .
\end{aligned}
$$

To estimate $\rho^{n+1}$, we use $z^{n+1}=R \Theta^{n+1}$ according to (47). Next, from Lemma 1 it follows that

$$
\left\|\rho^{n+1}\right\| \leq \Theta h^{m+1}\left\|\Theta\left(t_{n+1}\right)\right\|_{m+1}, \quad \forall t_{n+1} \in[0, T] .
$$

To estimate $\xi^{n+1}$, we introduce $\bar{z}^{n}$ that is obtained from $R z^{n}$, then from (51) it follows that

$$
z^{n+1}=E_{h} \bar{z}^{n}+E_{h}\left(z^{n}-\bar{z}^{n}\right)+\mathcal{E}_{2}+\mathcal{E}_{3}+\mathcal{E}_{4} .
$$

Next, taking into account the definition of $\xi$, we obtain from (28), (51) and with $\phi \in V_{h}$,

$$
\begin{aligned}
\xi^{n+1}= & E_{h} \bar{\xi}^{n}+E_{h}\left(z^{n}-\bar{z}^{n}\right)+S_{h} P_{0}\left(\left(z^{n+1}-\Theta^{n+1}\right)-\left(z^{n}-\Theta^{n}\left(\boldsymbol{X}_{h}^{n}\right)\right)\right)+ \\
& \Delta t S_{h} P_{0}\left(\frac{\Theta^{n+1}-\Theta^{n}\left(\boldsymbol{X}^{n}\right)}{\Delta t}-\left.\frac{D \Theta}{D \delta}\right|_{\delta=\delta^{\prime}}\right)+S_{h} P_{0}\left(\Theta^{n}\left(\boldsymbol{X}^{n}\right)-\Theta^{n}\left(\boldsymbol{X}_{h}^{n}\right)\right) \\
\equiv & \sum_{i=1}^{5} \mathcal{F}_{i}
\end{aligned}
$$

where $\bar{\xi}^{n}=\bar{z}^{n}-\Theta_{h}^{* n}$ is obtained from $\xi^{n}$. Now, we estimate the $\mathcal{F}_{i}$ 's terms in the $L^{2}$-norm.

\section{Estimate of $\mathcal{F}_{1}$ :}

From (41) and the definition of $E_{h}$, we have

$$
\left\|\mathcal{F}_{1}\right\| \leq\left\|\left|E_{h}\|\mid\| \bar{\xi}^{n}\|\leq\| \xi^{n} \| .\right.\right.
$$

\section{Estimate of $\mathcal{F}_{2}$ :}

$$
\left\|\mathcal{F}_{2}\right\| \leq\left\|\left|E_{h}\|\mid\| z^{n}-\bar{z}^{n} \|\right.\right.
$$


then, from the definition of $E_{h}$ and the triangle inequality, we have

$$
\begin{aligned}
\left\|\mathcal{F}_{2}\right\| & \leq\left\|\widehat{\Theta}^{n}-I_{m} \widehat{\Theta}^{n}\right\|+\left\|z^{n}-\widehat{\Theta}^{n}\right\|+\left\|I_{m} \widehat{\Theta}^{n}-\bar{z}^{n}\right\| \\
& \equiv \sum_{i=1}^{3} \mathcal{F}_{i}^{\prime}
\end{aligned}
$$

where $I_{m}$ is the polynomial interpolant of degree $m$ defined in (11). The approximation theory (12) and Lemma 1 yield to

$$
\mathcal{F}_{1}^{\prime} \leq K h^{m+1}\left|\Theta\left(t_{n}\right)\right|_{m+1}, \quad \forall t_{n} \in[0, T] .
$$

Then, by Lemma 4, we have

$$
\mathcal{F}_{2}^{\prime} \leq K h^{m+1}\left\|\Theta\left(t_{n}\right)\right\|_{m+1}, \quad \forall t_{n} \in[0, T] .
$$

To estimate $\mathcal{F}_{3}^{\prime}$, we obtain by using the same arguments as in [7]:

$$
\mathcal{F}_{3}^{\prime} \leq K h^{m+1}\left\|\Theta\left(t_{n}\right)\right\|_{m+1, \infty}, \quad \forall t_{n} \in[0, T] .
$$

Now, taking into account of the $\mathcal{F}_{i}^{\prime}$ estimates and that $W^{m+1, \infty}(\Omega) \subset H^{m+1}(\Omega)$ and $\left\|\Theta\left(t_{n}\right)\right\|_{m+1} \leq$ $K\left\|\Theta\left(t_{n}\right)\right\|_{m+1, \infty}$, it follows that

$$
\left\|\mathcal{F}_{2}\right\| \leq K h^{m+1}\left\|\Theta\left(t_{n}\right)\right\|_{m+1, \infty}, \quad \forall t_{n} \in[0, T] .
$$

\section{Estimate of $\mathcal{F}_{3}$ :}

We have

$$
\mathcal{F}_{3}=S_{h} P_{0} \int_{t_{n}}^{t_{n+1}} \frac{\partial\left(z(\boldsymbol{x}, t)-\Theta\left(\boldsymbol{X}_{h}\left(\boldsymbol{x}, t_{n+1} ; t\right), t\right)\right)}{\partial t} d t .
$$

Since $\left\|\left|S_{h} \|\right|<1\right.$ and $\|\left|P_{h} \|\right|$ are bounded, we obtain from Lemma 4

$$
\left\|\mathcal{F}_{3}\right\| \leq K h^{m+1}\left\|\Theta_{t}\right\|_{L^{2}\left(t_{n}, t_{n+1} ; H^{m+1}(\Omega)\right)} .
$$

\section{Estimate of $\mathcal{F}_{4}$ :}

By expanding in Taylor's series the term $\mathcal{F}_{4}$ along the trajectories $\boldsymbol{X}_{h}\left(\boldsymbol{x}, t_{n+1} ; t\right)$ with remaining integral, we obtain

$$
\frac{\Theta^{n+1}-\Theta^{n}\left(\boldsymbol{X}^{n}\right)}{\Delta t}-\frac{D \Theta}{D \delta}\left(\boldsymbol{X}\left(\boldsymbol{x}, t_{n+1} ; \delta^{\prime}\right), \delta^{\prime}\right)=\frac{1}{4 \Delta t} \int_{t_{n}}^{t_{n+1}}\left(t-t_{n}\right)\left(t-t_{n+1}\right) \frac{D^{3} \Theta\left(\boldsymbol{X}\left(\boldsymbol{x}, t_{n+1} ; t\right), t\right)}{D^{3} t} d t,
$$

next, by definition of $S_{h}$ and $P_{h}$, we obtain

$$
\left\|\mathcal{F}_{4}\right\|^{2} \leq K \frac{(\Delta t)^{4}}{4} \int_{t_{n}}^{t_{n+1}}\left\|\frac{D^{3} \Theta\left(\boldsymbol{X}\left(\boldsymbol{x}, t_{n+1} ; t\right), t\right)}{D^{3} t}\right\|^{2} d t
$$


which leads to

$$
\left\|\mathcal{F}_{4}\right\| \leq K \Delta t^{2}\left\|\frac{D^{3} \Theta}{D^{3} t}\right\|_{L^{2}\left(t_{n}, t_{n+1} ; L^{2}(\Omega)\right)}
$$

\section{Estimate of $\mathcal{F}_{5}$ :}

We have

$$
\Theta^{n}\left(\boldsymbol{X}^{n}\right)-\Theta^{n}\left(\boldsymbol{X}_{h}^{n}\right)=\left(\boldsymbol{X}^{n}-\boldsymbol{X}_{h}^{n}\right) \int_{0}^{1} D_{\boldsymbol{X}} \Theta^{n}\left(\boldsymbol{X}^{n}(\alpha)\right) d \alpha
$$

where $\boldsymbol{X}^{n}(\alpha)=\alpha \boldsymbol{X}^{n}+(1-\alpha) \boldsymbol{X}_{h}^{n}$, for $0<\alpha<1$. By using the definition of $S_{h}, P_{0}$ and from Lemma 3 , we obtain

$$
\left\|\mathcal{F}_{5}\right\| \leq K\left\|\boldsymbol{X}^{n}-\boldsymbol{X}_{h}^{n}\right\|\|\Theta\|_{L^{\infty}\left(t_{n}, t_{n+1} ; H^{1}(\Omega)\right)}
$$

then, by introducing (39) in the previous estimate, we have

$$
\left\|\mathcal{F}_{5}\right\| \leq K \Delta t\left(\left\|\boldsymbol{u}-\boldsymbol{u}_{h}\right\|_{L^{\infty}\left(0, T ; L^{2}(\Omega)\right)}+\mathcal{O}\left(\Delta t^{p}\right)\right)\|\Theta\|_{L^{\infty}\left(t_{n}, t_{n+1} ; H^{1}(\Omega)\right)} .
$$

Then, by substituting (43) in (56) we have

$$
\left\|\mathcal{F}_{5}\right\| \leq K \Delta t\left(\beta(t) h^{m+1}+\mathcal{O}\left(\Delta t^{p}\right)\right)\|\Theta\|_{L^{\infty}\left(t_{n}, t_{n+1} ; H^{1}(\Omega)\right)} .
$$

By summing the estimates $\mathcal{F}_{i}$ 's, we obtain

$$
\begin{aligned}
\left\|\xi^{n+1}\right\| \leq & \left\|\xi^{n}\right\|+K h^{m+1}\|\Theta\|_{m+1, \infty}+K \Delta t\left(\beta(t) h^{m+1}+\mathcal{O}\left(\Delta t^{p}\right)\right)+ \\
& \|\Theta\|_{L^{\infty}\left(t_{n}, t_{n+1} ; H^{1}(\Omega)\right)}+K h^{m+1}\left\|\Theta_{t}\right\|_{L^{2}\left(t_{n}, t_{n+1} ; H^{m+1}(\Omega)\right)}+K \Delta t^{2}\left\|\frac{D^{3} \Theta}{D^{3} t}\right\|_{L^{2}\left(t_{n}, t_{n+1} ; L^{2}(\Omega)\right)}
\end{aligned} .
$$

By using the Gronwall's inequality, we have

$$
\begin{aligned}
\left\|\xi^{n+1}\right\| \leq & \left\|\xi^{0}\right\|+K \frac{h^{m+1}}{\Delta t}\|\Theta\|_{L^{\infty}\left(0, T ; W^{m+1, \infty}(\Omega)\right)}+K\left(\max _{t \in(0, T)} \beta(t) h^{m+1}+\mathcal{O}\left(\Delta t^{p}\right)\right)\|\Theta\|_{L^{\infty}\left(0, T ; H^{1}(\Omega)\right)}+ \\
& K h^{m+1}\left\|\Theta_{t}\right\|_{L^{2}\left(0, T ; H^{m+1}(\Omega)\right)}+K \Delta t^{2}\left\|\frac{D^{3} \Theta}{D t^{3}}\right\|_{L^{2}\left(0, T ; L^{2}(\Omega)\right)}
\end{aligned}
$$

Hence, taking into account that $\xi^{0}=0$, and using (53), (54) and (57) together with the triangle inequality we obtain (52).

\section{Numerical results}

To examine the accuracy and performance of the proposed Galerkin-characteristics finite element method, we present numerical results for a coupled convection-diffusion-Darcy problem. Note that computations are carried out using triangular meshes with different element densities using the quadratic $P_{2}$ elements for all the solutions. For completeness, we formulate the corresponding basis functions for the 
approximation space $S_{h}$ in (3). Thus, using the standard mapping between the physical element with coordinates $(x, y)$ to the reference element with coordinates $(\xi, \eta)$, the six basis functions are defined by

$$
\begin{aligned}
& \phi_{1}(\xi, \eta)=(1-\xi-\eta)(1-2 \xi-2 \eta), \\
& \phi_{2}(\xi, \eta)=\xi(2 \xi-1), \\
& \phi_{3}(\xi, \eta)=\eta(2 \eta-1), \\
& \phi_{4}(\xi, \eta)=4 \xi(1-\xi-\eta), \\
& \phi_{5}(\xi, \eta)=4 \xi \eta \\
& \phi_{6}(\xi, \eta)=4 \eta(1-\xi-\eta) .
\end{aligned}
$$

The obtained linear systems of algebraic equations are solved using the conjugate gradient solver with

\begin{tabular}{|c|c|c|c|c|c|c|c|c|c|}
\hline \multirow[b]{3}{*}{$h$} & \multirow[b]{3}{*}{ Iter } & \multicolumn{6}{|c|}{$t=0.5$} & \multirow{2}{*}{\multicolumn{2}{|c|}{ Temperature $\Theta$}} \\
\hline & & \multicolumn{2}{|c|}{ Pressure $p$} & \multicolumn{2}{|c|}{ Velocity $u$} & \multicolumn{2}{|c|}{ Velocity $v$} & & \\
\hline & & $L^{2}$-error & rate & $L^{2}$-error & rate & $L^{2}$-error & rate & $L^{2}$-error & rate \\
\hline$\frac{1}{16}$ & 14.1 & 8.66439E-04 & - & 5.83217E-01 & - & 6.14833E-01 & - & $9.34698 \mathrm{E}-03$ & - \\
\hline$\frac{1}{32}$ & 13.3 & $2.35397 \mathrm{E}-04$ & 1.88 & $1.54118 \mathrm{E}-01$ & 1.92 & $1.61350 \mathrm{E}-01$ & 1.93 & $2.38584 \mathrm{E}-03$ & 1.97 \\
\hline$\frac{1}{64}$ & 12.2 & $6.26375 \mathrm{E}-05$ & 1.91 & 4.01657E-02 & 1.94 & 4.20505E-02 & 1.94 & $6.00609 \mathrm{E}-04$ & 1.99 \\
\hline$\frac{1}{128}$ & 11.1 & $1.60996 \mathrm{E}-05$ & 1.96 & $1.02524 \mathrm{E}-02$ & 1.97 & $1.06594 \mathrm{E}-02$ & 1.98 & 1.47062E-04 & 2.03 \\
\hline$\frac{1}{256}$ & 10.5 & 3.88779E-06 & 2.05 & $2.54539 \mathrm{E}-03$ & 2.01 & $8.52752 \mathrm{E}-03$ & 2.05 & $3.40665 \mathrm{E}-05$ & 2.11 \\
\hline
\end{tabular}
incomplete Cholesky decomposition. In addition, all stopping criteria for iterative solvers are set to $10^{-7}$

\begin{tabular}{|c|c|c|c|c|c|c|c|c|c|}
\hline & \multirow[b]{3}{*}{ Iter } & \multicolumn{6}{|c|}{$t=1$} & \multirow{2}{*}{\multicolumn{2}{|c|}{ Concentration $\Theta$}} \\
\hline & & \multicolumn{2}{|c|}{ Pressure $p$} & \multicolumn{2}{|c|}{ Velocity $u$} & \multicolumn{2}{|c|}{ Velocity $v$} & & \\
\hline & & $L^{2}$-error & rate & $L^{2}$-error & rate & $L^{2}$-error & rate & $L^{2}$-error & rate \\
\hline$\frac{1}{16}$ & 14.6 & $1.68771 \mathrm{E}-03$ & - & 6.93119E-01 & - & 7.45832E-01 & - & 1.27173E-02 & - \\
\hline$\frac{1}{32}$ & 13.8 & 4.61713E-04 & 1.87 & $1.81895 \mathrm{E}-01$ & 1.93 & $1.97089 \mathrm{E}-01$ & 1.92 & $3.29145 \mathrm{E}-03$ & 1.95 \\
\hline$\frac{1}{64}$ & 12.5 & $1.24573 \mathrm{E}-04$ & 1.89 & 4.70774E-02 & 1.95 & $5.06575 \mathrm{E}-02$ & 1.96 & 8.40153E-04 & 1.97 \\
\hline$\frac{1}{128}$ & 11.4 & $3.22415 \mathrm{E}-05$ & 1.95 & $1.20166 \mathrm{E}-02$ & 1.97 & $1.28412 \mathrm{E}-02$ & 1.98 & 2.12970E-04 & 1.98 \\
\hline$\frac{1}{256}$ & 10.9 & 8.00469E-06 & 2.01 & $3.02504 \mathrm{E}-03$ & 1.99 & $3.18812 \mathrm{E}-03$ & 2.01 & $5.25095 \mathrm{E}-05$ & 2.02 \\
\hline
\end{tabular}
which is small enough to guarantee that truncation errors in the algorithm dominate the total numerical errors.

Tableau 1. Relative $L^{2}$-error and convergence rates obtained for the pressure, velocity and concentration solutions in the accuracy test example of the coupled Darcy-transport problem at time $t=0.5$ and $t=1$. 


\subsection{Accuracy problem}

We demonstrate the accuracy for the proposed Galerkin-characteristics finite element method using the following time-dependent coupled convection-diffusion-Darcy equations

$$
\begin{aligned}
\nu(\Theta) \boldsymbol{u}+\nabla p & =(\Theta+f(x, y, t)) \boldsymbol{j}, & & (x, y, t) \in \Omega \times[0, T], \\
\nabla \cdot \boldsymbol{u} & =0, & & (x, y, t) \in \Omega \times[0, T], \\
\frac{\partial \Theta}{\partial t}+\boldsymbol{u} \cdot \nabla \Theta-\kappa \nabla^{2} \Theta & =g(x, y, t), & & (x, y, t) \in \Omega \times[0, T],
\end{aligned}
$$

equipped with the following initial condition

$$
\Theta(x, y, 0)=0 \quad(x, y) \in \Omega .
$$

In (58), $\boldsymbol{j}=(0,1)^{\top}$ is the unit vector in the upward direction and $\nu(\Theta)=\sin (\Theta)+2$. The functions $f(x, y, t)$ and $g(x, y, t)$ are calculated such that the exact solution of (58) is given by

$$
\begin{aligned}
& \boldsymbol{u}(x, y, t)=e^{-t / 4} \text { curl } \psi, \quad p(x, y, t)=(t+1) \cos (\pi x) \cos (\pi y), \\
& \Theta(x, y, t)=x^{2}(x-1)^{2} y^{2}\left(y-1^{2}\right) \sin (t)
\end{aligned}
$$

where the function $\psi$ is defined as

$$
\psi(x, y, t)=e^{-100\left((x-0.5)^{2}+(y-0.5)^{2}\right)} .
$$

A similar example has also been considered in [14] to validate an Eulerian finite element method. In our numerical simulations, the spatial domain $\Omega=[0,1] \times[0,1]$, the diffusion coefficient $\kappa=5 \times 10^{-4}$ and time step $\Delta t=0.05$. Table 1 summarizes the averaged number of iterations in the linear solver, the relative $L^{2}$-error and convergence rates at times $t=0.5$ and $t=1$ for the pressure $p$, the velocity $\boldsymbol{u}=(u, v)^{\top}$ and the temperature $\Theta$ using different structured meshes with uniform step $h$. It is clear that increasing the mesh density in the numerical simulations results in a decrease in the number of iterations needed for the linear solver and in the relative $L^{2}$-error for all variables and thus, a good approximation for pressure, velocity and temperature solutions at both considered instants. Furthermore, the proposed Galerkin-characteristics finite element method converges at about the same rate for all meshes and for all solutions confirming a second-order accuracy as expected.

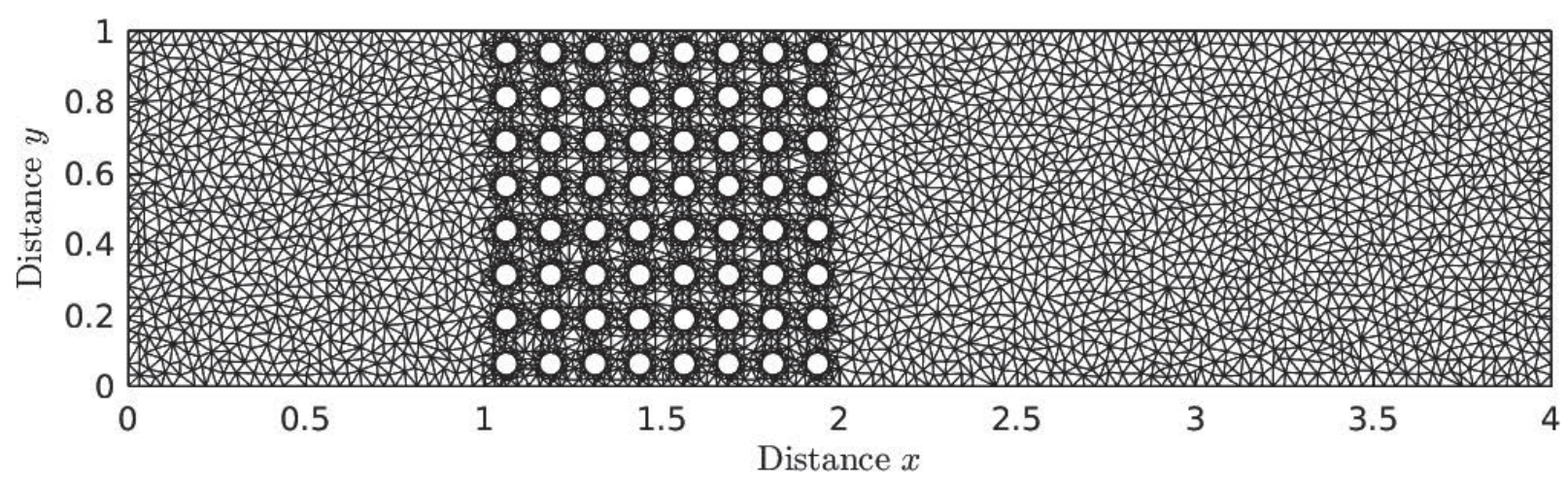

Figure 1. Mesh used in the simulations for the natural convection past an array of cylinders. 


\subsection{Natural convection past an array of cylinders}

In this example, we consider a natural convection problem in a channel past an array of circular cylinders. Here, we solve the equations (1) in a channel of length $L=4$ and height $H=1$ with 64 circular cylinders with equal diameter $D=0.0625$ uniformly distributed in the second quarter of the channel at the area $[1,2] \times[0,1]$. A similar computational domain has been investigated in [8] using the incompressible Navier-Stokes equations but for squared obstacles. In our computations, the source terms $\boldsymbol{f}=\mathbf{0}$ and $g=0$, and a nonlinear viscosity defined by $\nu(\Theta)=\Theta^{2}+2$ is used in (1). Here, the left and right vertical walls are respectively, at dimensionless temperatures $\Theta=0.5$ and $\Theta=-0.5$ whereas, the top and bottom walls are insulated. No-slip boundary condition is imposed at all cylinder walls and results are presented for the diffusion coefficient $\kappa=10^{-3}, 5 \times 10^{-4}$ and $10^{-4}$. Initially, the flow is at cold rest i.e., $\boldsymbol{u}=\mathbf{0}$ and $\Theta=-0.5$.

$$
\kappa=10^{-3}
$$
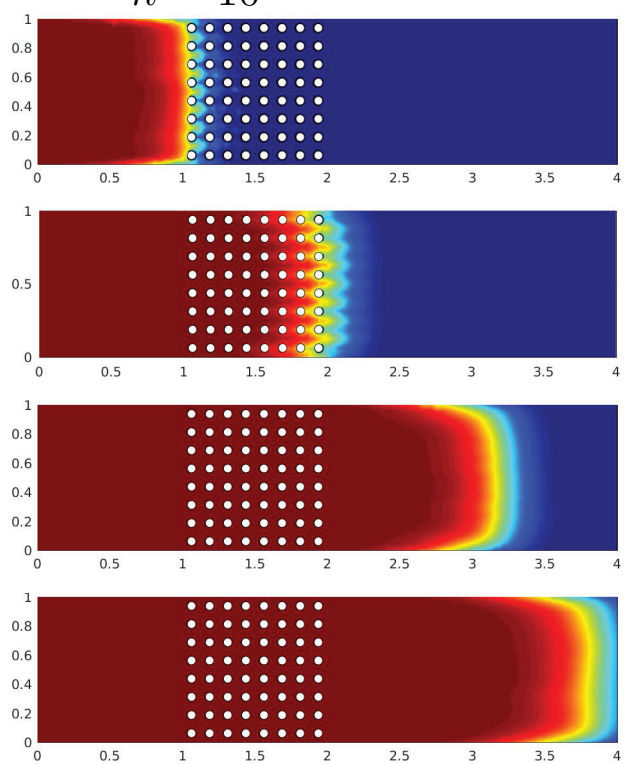

$$
\kappa=5 \times 10^{-4}
$$
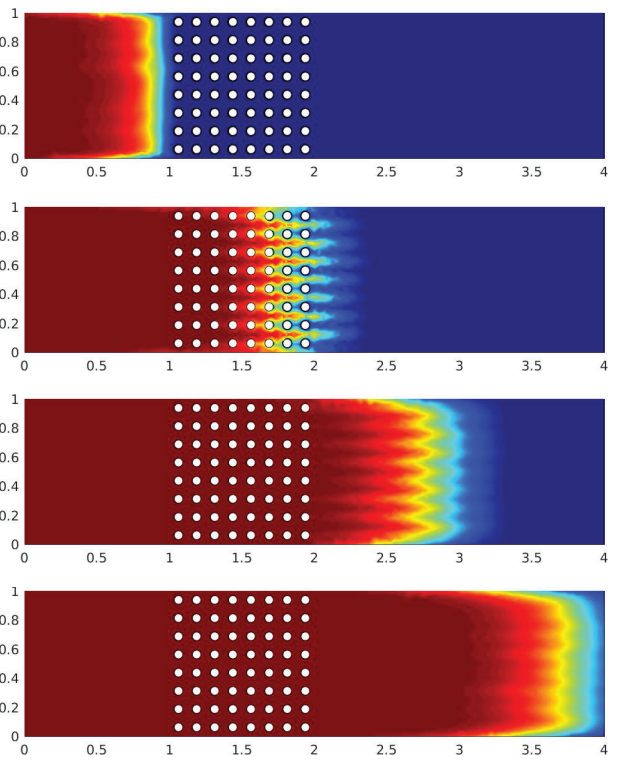

$\kappa=10^{-4}$
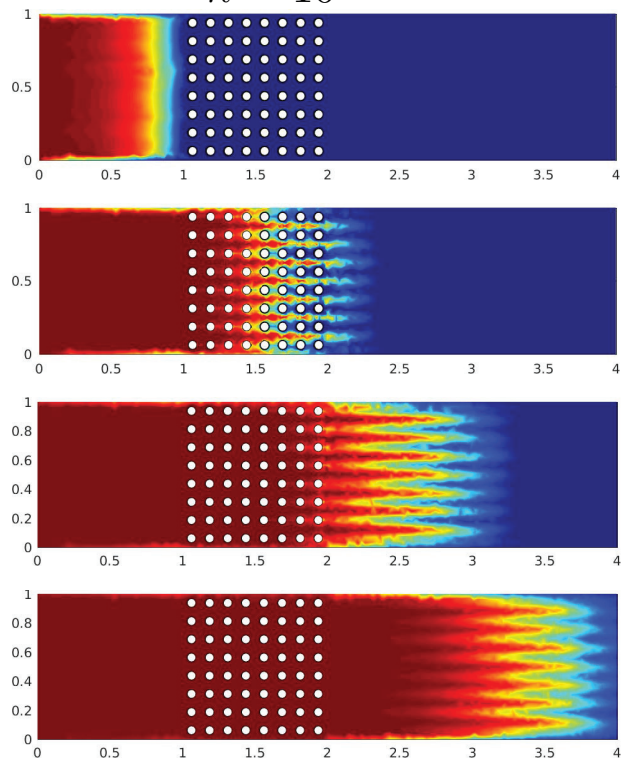

Figure 2. Temperature distributions obtained at $t=2$ (first row), $t=4$ (second row), $t=6$ (third row), and $t=8$ (fourth row) obtained for the natural convection past an array of cylinders using $\kappa=0.001$ (first column), $\kappa=0.0005$ (second column) and $\kappa=0.0001$ (third column).

Based on a mesh convergence study not reported here for brevity, the unstructured triangular mesh depicted in Figure 1 with 10911 elements and 24116 nodes is used in our simulations as it offers a compromise between accuracy and efficiency in the numerical method. Here, the quadratic $P_{2}$ elements are used to approximate the temperature, velocity and pressure in our Galerkin-characteristics finite element method. In Figure 2 and Figure 3, we display the results obtained for the temperature and velocity fields at four different instants namely, $t=2, t=4, t=6$ and $t=8$. To examine effects of diffusion in the moving thermal front past the cylinders, we present numerical results for three different cases with $\kappa=10^{-3}, 5 \times 10^{-4}$ and $10^{-4}$. It is clear that both the temperature patterns and velocity fields are influenced by the values taken by the diffusion coefficient $\kappa$. At earlier time of the simulation, the thermal front released from the left wall of the channel starts to develop and it is transported later on by the flow past the obstacles at downstream channel. Figure 2 indicates that the thermal fronts moving downstream past the cylinders at different speeds depending on the value of the diffusion coefficient $\kappa$ taken in the simulations. The results also indicate that as $\kappa$ decreases, the size of the transport speed increases with the thermal front exhibiting steep gradients with different magnitudes, thin boundary layer, 
and separating shear layers. It is worth remarking that for $\kappa=10^{-4}$, the problem becomes convectiondominated and steep fronts along with shock solutions are expected to appear in the temperature solution. We can see that the small complex structures of the temperature being captured by the proposed Galerkincharacteristics finite element method. Note that the performance of our method is very attractive since the computed solutions remain stable and highly accurate even when a relatively coarse mesh is used without requiring small time steps and mixed finite element discretizations or special pressure correction procedures.
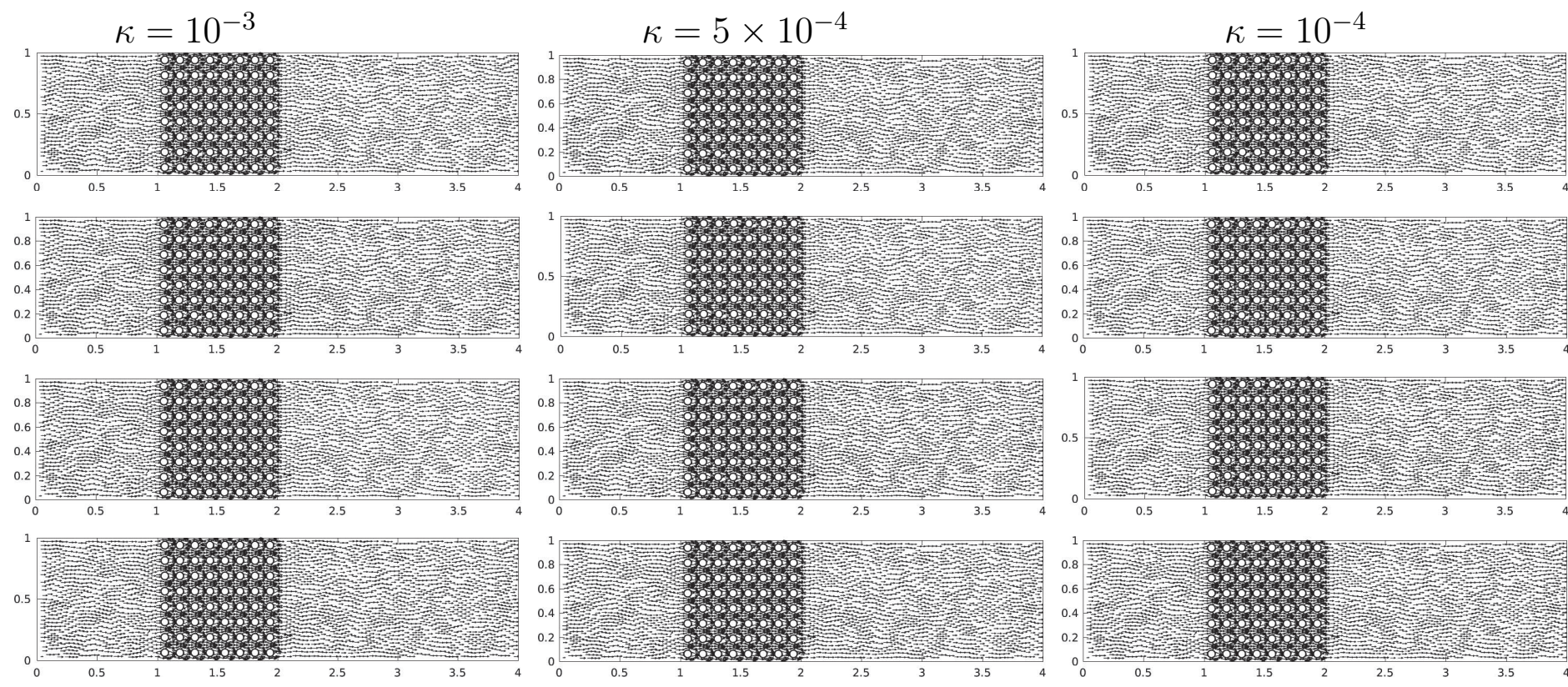

Figure 3. Velocity fields obtained at $t=2$ (first row), $t=4$ (second row), $t=6$ (third row), and $t=8$ (fourth row) obtained for the natural convection past an array of cylinders using $\kappa=0.001$ (first column), $\kappa=0.0005$ (second column) and $\kappa=0.0001$ (third column).
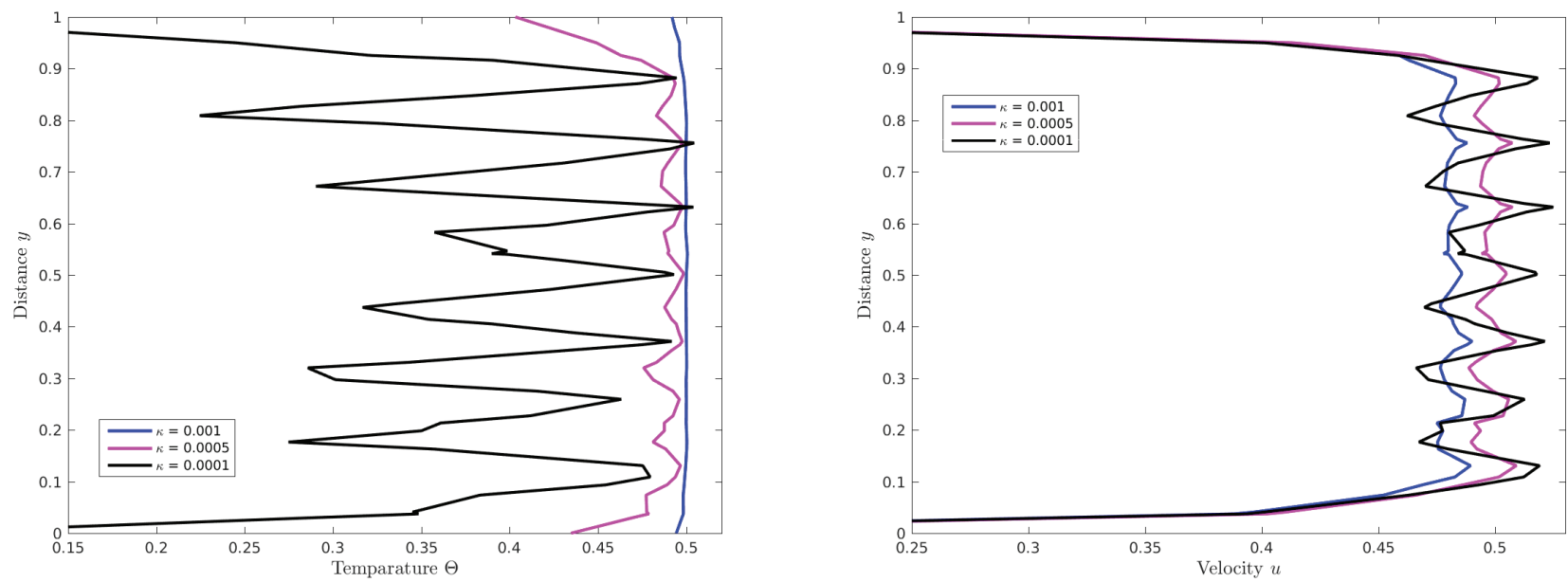

Figure 4. Cross-sections at $x=2.1$ of the temperature (left) and $u$-velocity (right) obtained for the natural convection past an array of cylinders at time $t=6$.

To further demonstrate these effects, we compare in Figure 4 vertical cross-sections at $x=2.1$ of the temperature and velocity $u$ at time $t=6$ for the considered values of the diffusion coefficient $\kappa$. It should also be pointed out that in our simulations for this problem, the number of iterations in the linear solver to reach the selected tolerance do not overpass 15 iterations for all considered mesh and values of $\kappa$. Taking into account this increase in the velocity field along with the considered values of 
$\kappa$, the flow appeared to change regimes. For the considered heat transfer conditions, it can be clearly seen that the complicated temperature and flow structures being well captured by the proposed Galerkincharacteristics finite element method. In fact, the computed solutions reveal the physics well in this test example for coupled Darcy-transport problems. It should be stressed that the performance of the proposed method is very attractive since the computed solutions remain stable and highly accurate even when coarse meshes are used without requiring nonlinear solvers or small time steps to be taken in the simulations. In addition, the presented results clearly indicate that this method is suited for prediction of natural convection in porous media.

\section{Conclusions}

In the current work, we have presented analysis of a Galerkin-characteristics finite element for solving convection-diffusion problems in porous media. The governing equations consist of a nonlinear Darcy problem for the flow field and pressure coupled with a time-dependent convection-diffusion equation for the temperature. The coupled system has been integrated using a Galerkin-characteristic finite element method which combines the semi-Lagrangian method for the time integration with the Galerkin finite element method for the space discretization on unstructured grids. A study of stability and convergence have been then demonstrated and an optimal a priori error estimate has been established for the proposed method. The proposed Galerkin-characteristics finite element allows for the same finite element space to be used for all solutions to the problem including the pressure, velocity and temperature. As a consequence, the proposed method avoids the mixed finite element discretizations which require more computational cost related to the mesh generation and the element matrix assembly. Furthermore, the proposed Galerkin-characteristic finite element method is suitable for complex geometries, independent of the sizes and arrangement of the mesh elements, and it can easily combine different polynomial orders of elements. Numerical results have been presented for an accuracy test example with known analytical solutions. The method has also been applied for solving a natural convection problem past an array of cylinders in porous media and the obtained results exhibited good shape, high accuracy and stability behavior for all transport regimes considered. In addition, the presented results demonstrate the capability of the proposed Galerkin-characteristic finite element method that can provide insight to complex coupled nonlinear Darcy-transport features. Future work will concentrate on the extension of this method to convection-diffusion problems in three-dimensional porous media using high-order unified finite element discretizations on unstructured meshes.

\section{References}

[1] T. Allen and M. Zerroukat. A semi-Lagrangian semi-implicit immersed boundary method for atmospheric flow over complex terrain. Journal of Computational Physics, 397:108857, 2019.

[2] Y. Amanbek, G. Singh, G. Pencheva, and M.F. Wheeler. Error indicators for incompressible Darcy flow problems using enhanced velocity mixed finite element method. Computer Methods in Applied Mechanics and Engineering, 363:112884, 2020.

[3] J. Bear. Hydraulics of groundwater. Springer-Verlag, New York, NY, USA, 1979.

[4] J. Bear. Dynamics of fluids in porous media. Courier Corporation, 2013.

[5] J. Bear, J.M. Buchlin, et al. Modelling and applications of transport phenomena in porous media, volume 5. Springer, 1991. 
[6] M. Bermejo, R. El-Amrani. A finite element semi-Lagrangian explicit Runge-Kutta-Chebyshev method for convection dominated reaction-diffusion problems. J. Comp. Applied Math., 154:27-61, 2003.

[7] R. Bermejo and M. El Amrani. Lagrangian explicit Runge-Kutta-Chebyshev method for convection dominated reaction-diffusion problems. J. Comp. Appl. Mat., 154, 27-61, 2003.

[8] J. Bernsdorf, F. Durst, and M. Schäfer. Comparison of cellular automata and finite volume techniques for simulation of incompressible flows in complex geometries. Int. J. Numer. Meth. Fluids, 29:251-264, 1999.

[9] C.R. Bochev, P.B. Dohrmann. A computational study of stabilized, low-order $C^{0}$ finite element approximations of Darcy equations. Computational Mechanics., 38:223-323, 2006.

[10] P.B. Bochev, C.R. Dohrmann, and M.D. Gunzburger. Stabilization of low-order mixed finite elements for the Stokes equations. SIAM Journal on Numerical Analysis, 44(1):82-101, 2006.

[11] J.M. Boland and R. A. Nicolaides. Stable and semistable low order finite elements for viscous flows. SIAM J. Numer. Anal., 22:474-492, 1985.

[12] F. Brezzi. On existence, uniqueness, and approximation of saddle-point problems arising from Lagrangian multipliers. RAIRO Model. Math. Anal. Numer., 21: 129-151, 1974.

[13] J. Capecelatro. A purely Lagrangian method for simulating the shallow water equations on a sphere using smooth particle hydrodynamics. Journal of Computational Physics, 356:174-191, 2018.

[14] N. Chalhoub, P. Omnes, T. Sayah, and R. El-Zahlaniyeh. Full discretization of time dependent convection-diffusionreaction equation coupled with the Darcy system. Calcolo, 57:4, 2019.

[15] Z. Chen and R. Ewing. Mathematical analysis for reservoir models. SIAM J. Math. Anal., pages 30, 431-453, 1999.

[16] G. De Marsily. Quantitative hydrogeology. Groundwater Hydrology for Engineers; Academic Press: New York, NY, USA, 1986.

[17] M. Dejam and H. Hassanzadeh. Diffusive leakage of brine from aquifers during $\mathrm{CO}_{2}$ geological storage. Advances in Water Resources, 111:36-57, 2018.

[18] C.R. Dohrmann and P.B. Bochev. A stabilized finite element method for the Stokes problem based on polynomial pressure projections. International Journal for Numerical Methods in Fluids, 46:183-201, 2004.

[19] T.F. Douglas, J. Russell. Numerical methods for convection dominated diffusion problems based on combining the method of characteristics with finite elements or finite differences. SIAM J. Numer. Anal., 19:871-885, 1982.

[20] M. El-Amrani and M. Seaid. Convergence and stability of finite element modified method of characteristics for the incompressible Navier-Stokes equations. J. Numer. Math., 15:101-135, 2007.

[21] M. El-Amrani and M. Seaid. Numerical simulation of natural and mixed convection flows by Galerkin-characteristic method. Int. J. Numer. Meth. Fluids., 53(12):1819-1845, 2007.

[22] M. El-Amrani and M. Seaid. Numerical simulation of natural and mixed convection flows by Galerkin-characteristic method. Int. J. Num. Meth. Fluids., 53:1819-1845, 2007.

[23] M. El-Amrani and M. Seaid. A finite element modified method of characteristics for convective heat transport. $\mathrm{Nu}$ merical Methods for Partial Differential Equations, 24(3):776-798, 2008.

[24] M. El-Amrani and M. Seaid. An essentially non-oscillatory semi-Lagrangian method for tidal flow simulations. Int. J. Numer. Meth. Engng., 81(7):805-834, 2010.

[25] M. El-Amrani and M. Seaid. An L ${ }^{2}$-projection for the Galerkin-characteristic solution of incompressible flows. SIAM Journal on Scientific Computing, 33(6):3110-3131, 2011.

[26] V. Faraoni, F. Atieh, and S. Dussault. Cosmological analogies, Lagrangians, and symmetries for convective-radiative heat transfer. European Physical Journal C 80, Springer, page 706, 2020. 
[27] X. Feng. On existence and uniqueness results for a coupled system modeling miscible displacement in porous media. J. Math. Anal. Appl., pages 194, 883-910, 1995.

[28] C. Foicas, C. Guillopé, and R. R. Temam. Lagrangian representation of the flow. J. Diff. Eqns, 57:440-449, 1985.

[29] K. Ghesmat, H. Hassanzadeh, and J. Abedi. The effect of anisotropic dispersion on the convective mixing in long-term CO2 storage in saline aquifers. AIChE J., 57:561-570, 2011.

[30] M. Gunzburger. Finite element methods for viscous incompressible flows. Academic Press, Boston, 1989.

[31] A. Halassi, J. Joundy, L. Salhi, and A. Taik. A meshfree method for heat explosion problems with natural convection in inclined porous media. MATEC Web of Conferences, 241, 01019, 2018.

[32] M. Hennessey, A.K. Kapila, and D.W. Schwendeman. An HLLC-type riemann solver and high-resolution Godunov method for a two-phase model of reactive flow with general equations of state. Journal of Computational Physics, 405:109180, 2020.

[33] J.G. Heywood and R. Rannacher. Finite element approximation of the non-stationary Navier-Stokes problem: Part III. SIAM Journal of Numerical Analysis., 25, 489-512, 1988.

[34] P. Houston, S. Roggendorf, G. Kristoffer, and D. Zee. Eliminating Gibbs phenomena: A non-linear Petrov-Galerkin method for the convection-diffusion-reaction equation. Computers and mathematics with applications, 80:851-873, 2020 .

[35] V. Maz’ya. Sobolev spaces. Springer, 2013.

[36] H. Notsu, H. Rui, and M. Tabata. Development and L2-analysis of a single-step characteristics finite difference scheme of second order in time for convection-diffusion problems. J. Algorithms Comput. Technol., 7:343-380, 2013.

[37] H. Notsu and M. Tabata. Error estimates of a pressure-stabilized characteristics finite element scheme for the Oseen equations. J. Sci. Comput., 65:940-955, 2015.

[38] O. Pironneau. On the transport-diffusion algorithm and its applications to the Navier-Stokes equations. Numer. Math., 38:309-332, 1982.

[39] J. Pudykiewicz and A. Staniforth. Some properties and comparative performance of the semi-Lagrangian method of robert in the solution of the advection-diffusion equation. Atmosphere-Ocean, 22:3:283-308, 1984.

[40] A. Robert. A stable numerical integration scheme for the primitive meteorological equations. Atmos. Ocean., 19:3546, 1981.

[41] H. Rui and J. Zhang. A stabilized mixed finite element method for coupled Stokes and Darcy flows with transport. Computer Methods in Applied Mechanics and Engineering, 315:169-189, 2017.

[42] L. Salhi, M. El-Amrani, and M. Seaid. A Galerkin-characteristic unified finite element method for moving thermal fronts in porous media. Journal of Computational and Applied Mathematics, page 113159, 2020.

[43] L. Salhi, M. El-Amrani, and M. Seaid. A stabilized semi-Lagrangian finite element method for natural convection in Darcy flows. Computational and Mathematical Methods, page e1140, 2020.

[44] M. Seaid. Semi-Lagrangian integration schemes for viscous flows. Comp. Methods in App. Math., 4:392-409, 2002.

[45] J.F. Steven. Semi-Lagrangian Advection Methods and Their Applications in Geoscience. Elsevier, 2019.

[46] E. Süli. Convergence and nonlinear stability of the Lagrange-Galerkin method for the Navier-stokes equations. Numer. Math., 53:1025-1039, 1988.

[47] D. Sun, Q. Guo, C Li, and P. Liu. Assessment of optimized symmetric fourth-order weighted essentially nonoscillatory scheme in direct numerical simulation of compressible turbulence. Computers \& fluids, 197:104383, 2020.

[48] A. Temperton, C. Staniforth. An efficient two-time-level semi-Lagrangian semi-implicit integration scheme. Quart. J. Roy. Meteor. Soc., 113:1025-1039, 1987.

[49] S. Yin. A fully coupled finite element framework for thermal fracturing simulation in subsurface cold $\mathrm{CO}_{2}$ injection. Petroleum, 4:65-74, 2018. 